\title{
Strong-electric-field eigenvalue asymptotics for the Iwatsuka model
}

AUTHOR(S):

Shirai, S

CITATION:

Shirai, S. Strong-electric-field eigenvalue asymptotics for the Iwatsuka model. JOURNAL OF MATHEMATICAL PHYSICS 2005, 46(5): 052112.

ISSUE DATE:

2005-05

URL:

http://hdl.handle.net/2433/50503

\section{RIGHT:}

Copyright 2005 American Institute of Physics. This article may be downloaded for personal use only. Any other use requires prior permission of the author and the American Institute of Physics. 


\title{
Strong-electric-field eigenvalue asymptotics for the Iwatsuka model
}

\author{
Shin-ichi Shiraia) \\ Research Institute for Mathematical Sciences, Kyoto University, Sakyo, \\ Kyoto 606-8502, Japan
}

(Received 6 May 2004; accepted 3 March 2005; published online 5 May 2005)

We consider the two-dimensional Schrödinger operator, $H_{g}(b)=-\partial^{2} / \partial x^{2}$ $+[(1 / \sqrt{-1})(\partial / \partial y)-b(x)]^{2}-g V(x, y)$, where $V$ is a non-negative scalar potential decaying at infinity like $(1+|x|+|y|)^{-m}$, and $(0, b(x))$ is a magnetic vector potential. Here, $b$ is of the form $b(x)=\int_{0}^{x} B(t) \mathrm{d} t$ and the magnetic field $B$ is assumed to be positive, bounded, and monotonically increasing on $\mathbf{R}$ (the Iwatsuka model). Following the argument as in Refs. 15, 16, and 17 [Raikov, G. D., Lett. Math. Phys., 21, 41-49 (1991); Raikov, G. D, Commun. Math. Phys., 155, 415-428 (1993); Raikov, G. D. Asymptotic Anal., 16, 87-89 (1998)], we obtain the asymptotics of the number of discrete spectra of $H_{g}(b)$ crossing a real number $\lambda$ in the gap of the essential spectrum as the coupling constant $g$ tends to $\pm \infty$, respectively. (C) 2005 American Institute of Physics. [DOI: 10.1063/1.1897844]

\section{INTRODUCTION}

We consider the two-dimensional Schrödinger operator with electromagnetic field

$$
H_{g}(b)=-\frac{\partial^{2}}{\partial x^{2}}+\left(\frac{1}{\sqrt{-1}} \frac{\partial}{\partial y}-b(x)\right)^{2}-g V(x, y) .
$$

Here, $V(x, y)$ is a scalar potential decaying at infinity and $(0, b(x))$ is a magnetic vector potential given by the form $b(x)=\int_{0}^{x} B(t) \mathrm{d} t$ for a positive magnetic field $B$, which depends only on the variable $x$ of $(x, y) \in \mathbf{R}^{2}$.

The purpose of this paper is to investigate the number of discrete spectra of $H_{g}(b)$ crossing a real number $\lambda$ in the gap of the essential spectrum as the coupling constant $g$ tends to $\pm \infty$, respectively (the precise formulation is given below).

We fix some notations. We denote the set of all integers by $\mathbf{Z}$ and denote the set of nonnegative integers by $\mathbf{N}$. We denote the cardinal number of set $A$ by $\sharp A$. We denote both $\partial / \partial x$ and $\mathrm{d} / \mathrm{d} x$ by $\partial_{x}$, etc. We denote by $C^{k}\left(\Omega, \Omega^{\prime}\right)$ the set of all $\Omega^{\prime}$-valued, $C^{k}$-functions on $\Omega$, and by $C_{0}^{\infty}(\Omega)$ the set of all compactly supported, smooth functions on $\Omega$. We use $|\cdot|$ to denote the Euclidean norms and use the notations $\langle z\rangle=\left(1+|z|^{2}\right)^{1 / 2}$ for any $z \in \mathbf{R}^{n}$ and $\langle x ; y\rangle=\left(1+|x|^{2}\right.$ $\left.+|y|^{2}\right)^{1 / 2}$ for any $(x, y) \in \mathbf{R}^{n} \times \mathbf{R}^{m}$. We denote by $Q(z, r)$ the open cube of radius $r$, centered at $z$, with sides parallel to the coordinate axes. We denote by $\operatorname{Spec}(A)$ the spectrum of any self-adjoint operator $A$, and by $N(\alpha<A<\beta)$ the dimension of the range of the spectral projection for $A$ on the interval $(\alpha, \beta)$. The notations $N(A>\alpha), N(A<\beta)$, etc., are defined similarly.

To formulate our results we make the following assumptions for the magnetic field $B$ and the electric potential $V$.

(B.1) The magnetic field $B$ is a real-valued, smooth and monotonically increasing function on $\mathbf{R}$. Moreover, there exist positive numbers $B_{ \pm}$such that $B_{-}<B_{+}$, and $\lim _{x \rightarrow \pm \infty} B(x)=B_{ \pm}$hold, respectively.

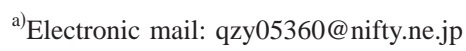


(B.2) In addition to (B.1), there exists a positive number $M$ such that, for any multi-index $\alpha$, the estimate $\left|\partial_{x}^{\alpha}\left(B(x)-B_{ \pm}\right)\right| \leqslant C_{\alpha}\langle x\rangle^{-M}$ holds for $\pm x \geqslant 0$, respectively. Here, the constant $C_{\alpha}$ is independent of $x$, and $B_{ \pm}$are as in (B.1).

(V.1) The scalar potential $V$ is a non-negative smooth function on $\mathbf{R}^{2}$. Moreover, there exists a positive number $m$ such that, for any multi-index $\alpha$, the estimate $\left|\partial_{z}^{\alpha} V(z)\right| \leqslant C_{\alpha}\langle z\rangle^{-m-|\alpha|}$ holds for all $z \in \mathbf{R}^{2}$. Here, the constant $C_{\alpha}$ is independent of $z$.

(V.2) There exists a positive number $C$ such that the estimate $V(z) \geqslant C\langle z\rangle^{-m}$ holds for all $z \in \mathbf{R}^{2}$.

(V.3) Let $(r, \omega) \in[0, \infty) \times S^{1}$ be the polar coordinates of $z \in \mathbf{R}^{2}$, i.e., $r=|z|$ and $\omega=z /|z|$. There exists a measurable, bounded and positive function $v$ on the unit circle $S^{1}$ such that $\lim _{r \rightarrow \infty} r^{m} V(z)=v(\omega)$ holds for any $\omega=z /|z| \in S^{1}$.

(V.4) In addition to (V.1), we have the limit

$$
\lim _{\varepsilon \downarrow 0} \limsup _{\mu \downarrow 0} \mu^{2 / m} \operatorname{Vol}\left\{z \in \mathbf{R}^{2} \mid(1-\varepsilon) \mu<V(z)<(1+\varepsilon) \mu\right\}=0 .
$$

Here, "Vol" stands for the Euclidean volume, and $m$ is as in (V.1).

Under the assumptions $(B .1)$ and $(V .1)$, the operator $H_{g}(b)$ is essentially self-adjoint on $C_{0}^{\infty}\left(\mathbf{R}^{2}\right)$ for any $g \in \mathbf{R}$ [see Avron, Herbst, and Simon (1978)]. In what follows we identify any closable operator with its operator closure if there is no fear of confusion. Iwatsuka (1985) investigated the spectral properties of the unperturbed operator $H_{0}(b)$, which is called the Iwatsuka model by some authors [Mantoiu and Purice (1997), Exner and Kovařik (1999), and Shirai (2003)]. Iwatsuka's result says that, under $(B .1)$, the spectrum of $H_{0}(b)$ is absolutely continuous and $\operatorname{Spec}\left(H_{0}(b)\right)=\cup_{n=0}^{\infty}\left[\Lambda_{n}^{-}, \Lambda_{n}^{+}\right]$holds, where we set $\Lambda_{n}^{ \pm}=(2 n+1) B_{ \pm}$for any $n \in \mathbf{N}$, respectively, and for notational convenience, we set $\Lambda_{-1}^{+}=\Lambda_{-1}^{-}=0$.

Under $(B .1)$ and $(V .1)$, the multiplication operator $V$ is relatively compact with respect to $H_{0}(b)$, so the essential spectrum of $H_{g}(b)$ coincides with that of $H_{0}(b)$ for any $g$ [see, e.g., Reed and Simon (1978, Sec. XIII.4)]. In particular, the operator $H_{g}(b)$ may have discrete spectra (i.e., discrete eigenvalues of finite multiplicity) in the gaps of the essential spectrum.

We make some additional notations. Let $v$ be as in (V.3). Set $S_{ \pm}^{1}=\left\{z=(x, y) \in \mathbf{R}^{2}|| z \mid=1, \pm x\right.$ $\geqslant 0\}$ and set $\hat{v}_{ \pm}=\int_{S_{ \pm}^{1}}(\omega)^{2 / m} \mathrm{~d} \omega$, respectively. For any $\lambda \in \mathbf{R} \backslash \operatorname{Spec}\left(H_{0}(b)\right)$ and for any $l \in \mathbf{N}$, set

$$
\nu_{l}(\lambda)=\frac{1}{4 \pi}\left(B_{+} \hat{v}_{+}\left|\Lambda_{l}^{+}-\lambda\right|^{-2 / m}+B_{-} \hat{v}_{-}\left|\Lambda_{l}^{-}-\lambda\right|^{-2 / m}\right) .
$$

For any interval $[\lambda, \mu] \subset \mathbf{R} \backslash \operatorname{Spec}\left(H_{0}(b)\right)$ and for any $l \in \mathbf{N}$, set

$$
\nu_{l}([\lambda, \mu])=\frac{1}{2 \pi m}\left(B_{+} \hat{v}_{+} \int_{\lambda}^{\mu}\left|\Lambda_{l}^{+}-t\right|^{-2 / m-1} \mathrm{~d} t+B_{-} \hat{v}_{-} \int_{\lambda}^{\mu}\left|\Lambda_{l}^{-}-t\right|^{-2 / m-1} \mathrm{~d} t\right) .
$$

If we assume that $0<\Lambda_{n}^{+}<\lambda<\mu<\Lambda_{n+1}^{-}$for some $n \in \mathbf{N}$, the sums $\Sigma_{l \in \mathbf{N}} \nu_{l}(\lambda)$ and $\Sigma_{l \in \mathbf{N}} \nu_{l}([\lambda, \mu])$ converge when $0<m<2$, and the relation $\nu_{l}([\lambda, \mu])=\nu_{l}(\mu)-\nu_{l}(\lambda)$ holds when $l$ $\geqslant n+1$.

We denote by $N_{g}^{ \pm}(\lambda)$ the number of eigenvalues of $H_{ \pm g^{\prime}}(b)$ crossing $\lambda$ as $g^{\prime}$ increases from zero to $g$, i.e., $N_{g}^{ \pm}(\lambda)=\Sigma_{0<g^{\prime}<g} \operatorname{dim} \operatorname{Ker}\left(H_{ \pm g^{\prime}}(b)-\lambda\right)$, respectively. Note that the sum above is meaningful under the assumptions $(B .1)$ and $(V .2)$, since the standard perturbation theory shows that for every fixed $g>0$ the set of values of $g^{\prime} \in(0, g)$ for which $\operatorname{dim} \operatorname{Ker}\left(H_{ \pm g^{\prime}}(b)-\lambda\right)$ is not equal to zero is finite [see, e.g, Reed and Simon (1978)].

The main results in this paper are the following.

Theorem 1.1: Let $\Lambda_{n-1}^{+}<\lambda<\Lambda_{n}^{-}$for some $n \in \mathbf{N}$. Assume that (B.1), (B.2), (V.1)-(V.4) hold. Moreover, assume that the constant $m$ in (V.1) satisfies $0<m<2$. Then we have

$$
\lim _{g \rightarrow \infty} g^{-2 / m} N_{g}^{+}(\lambda)=\sum_{l \geqslant n} \nu_{l}(\lambda)
$$

Remark 1.2: In fact, the conclusion of Theorem 1.1 is still valid under weaker conditions on 
$B$ and $V$ in the case of $\lambda<\Lambda_{0}^{-}$. See Lemma 3.8 in Sec. III below.

Corollary 1.3: Let $\Lambda_{n-1}^{+}<\lambda<\mu<\Lambda_{n}^{-}$for some $n \in \mathbf{N}$. Under the same assumption as in Theorem 1.1, we have $\lim _{g \rightarrow \infty} g^{-2 / m} N\left(\lambda \leqslant H_{g}(b)<\mu\right)=\Sigma_{l \geqslant n} \nu_{l}([\lambda, \nu])$.

Theorem 1.4: Let $\Lambda_{n}^{+}<\lambda<\Lambda_{n+1}^{-}$for some $n \in \mathbf{N}$. Assume that (B.1), (B.2), (V.1)-(V.4) hold. Then we have $\lim _{g \rightarrow \infty} g^{-2 / m} N_{g}^{-}(\lambda)=\Sigma_{0 \leqslant l \leqslant n} \nu_{l}(\lambda)$.

Theorem 1.5: Let $\Lambda_{n-1}^{+}<\lambda<\mu<\Lambda_{n}^{-}$for some $n \in \mathbf{N}$. Assume that (B.1), (B.2), (V.1)-(V.4) hold. Moreover, assume that the constant $m$ in (V.1) satisfies $m>2$. Then we have $\liminf _{g \rightarrow \infty} g^{-2 / m} N\left(\lambda \leqslant H_{g}(b)<\mu\right) \geqslant \sum_{l \geqslant n} \nu_{l}([\lambda, \mu])$.

At present, the author has not obtained the upper estimate for $N\left(\lambda \leqslant H_{g}(b)<\mu\right)$ in the case of $m>2$, nor the results for the case of $m=2$.

Remark 1.6:

(1) The study of eigenvalues in the spectral gaps of the Schrödinger operators has a long history [see, e.g., Birman (1991), Alama, Deift, and Hempel (1989), Hempel and Levendorskiŭ (1998) and references therein]. In the case of constant magnetic fields (i.e., the case of $B_{+}$ $=B_{-}$in our notation), Raikov $(1991,1993)$ has obtained the strong-electric-field asymptotics as in Theorem 1.1 and Corollary 1.3 above when the scalar potential decays slowly at infinity (i.e., the case of $0<m \leqslant 2$ in our situation). Moreover, for a class of nonconstant magnetic fields which includes the Iwatsuka model, Raikov $(1998,1993)$ shows that the asymptotics of $N_{g}^{+}(\lambda)$ are Weylian, i.e., the asymptotic relation $\lim _{g \rightarrow \infty} g^{-1} N_{g}^{+}(\lambda)$ $=(1 / 4 \pi) \int_{\mathbf{R}^{2}} V(x, y) \mathrm{d} x \mathrm{~d} y$ holds when the scalar potential decays rapidly (the case of $m>2$ in our situation).

(2) Hempel and Levendorskiŭ (1998) [see also Levendorskiŭ (1995, 1996)] study the asymptotics of $N_{g}^{ \pm}(\lambda)$ for the magnetic Schrödinger operators $H-g V=(-\sqrt{-1} \nabla-\mathbf{a})^{2}+W-g V$ on $L^{2}\left(\mathbf{R}^{n}\right)$ under rather general conditions on $\mathbf{a}, W$, and $V$. Especially, $V$ is not assumed to have a definite sign. They assume, however, the existence of the density of states $\rho(\lambda, H)$ $=\lim _{R \rightarrow \infty} N\left(\left.H\right|_{Q_{R}}<\lambda\right) / \operatorname{Vol}\left(Q_{R}\right)$ for the unperturbed operator $H$, for which the asymptotic coefficient as in Theorem 1.1 is expressed as

$$
\lim _{g \rightarrow \infty} g^{-n / m} N_{g}^{+}(\lambda)=\int_{\mathbf{R}^{n}} \mathrm{~d} z \int_{\lambda}^{\lambda+v(\omega) r^{-m}} \mathrm{~d} \rho(t, H)
$$

when $V(z)=v(\omega) r^{-m}$ at infinity and $0<m<2$, for example. Here, $Q_{R}$ is a cube of side length $R$ and we denote by $\left.H\right|_{Q_{R}}$ the Dirichlét realization of $H$ on $Q_{R}$. On the contrary, the Iwatsuka model has no canonical density of states because of lack of spacial symmetry of the magnetic field B. Thus, at least, we need modify their argument. Indeed, although the isotropic density of states $\rho\left(\lambda, H_{0}(b)\right)=\left(B_{+} / 4 \pi\right) \#\left\{l \in \mathbf{N} \mid \Lambda_{l}^{+}<\lambda\right\}+\left(B_{-} / 4 \pi\right) \#\left\{l \in \mathbf{N} \mid \Lambda_{l}^{-}<\lambda\right\}$ for the Iwatsuka model do exist under the assumption in Theorem 1.1, the quantity

$$
\int_{\mathbf{R}^{2}} \mathrm{~d} x \mathrm{~d} y \int_{\lambda}^{\lambda+v(\omega) r^{-m}} \mathrm{~d} \rho\left(t, H_{0}(b)\right)=\frac{B_{+}}{4 \pi} \frac{\hat{v}}{2} \sum_{l \geqslant n+1}\left|\Lambda_{l}^{+}-\lambda\right|^{-2 / m}+\frac{B_{-}}{4 \pi} \frac{\hat{v}}{2} \sum_{l \geqslant n+1}\left|\Lambda_{l}^{-}-\lambda\right|^{-2 / m},
$$

where $\hat{v}=\int_{S^{1}} v(\omega)^{2 / m} \mathrm{~d} \omega$, does not give the correct asymptotic coefficient (1.1), which can be expressed as

$$
\int_{\{x \geqslant 0\}} \mathrm{d} x \mathrm{~d} y \int_{\lambda}^{\lambda+v(\omega) r^{-m}} \mathrm{~d} \rho_{+}\left(t, H_{0}(b)\right)+\int_{\{x \leqslant 0\}} \mathrm{d} x \mathrm{~d} y \int_{\lambda}^{\lambda+v(\omega) r^{-m}} \mathrm{~d} \rho_{-}\left(t, H_{0}(b)\right),
$$

where $\rho_{ \pm}\left(t, H_{0}(b)\right)=\left(B_{ \pm} / 4 \pi\right) \#\left\{l \in \mathbf{N} \mid \Lambda_{l}^{ \pm}<t\right\}$, respectively.

The organization of this paper is as follows: Sec. II contains some preliminary results from functional analysis and the theory of pseudodifferential operators. In Sec. III, we give a proof of Theorem 1.1 in the case where the control point $\lambda$ is fixed below the infimum of the essential spectrum of $H_{g}(b)$. In Sec. IV, we recall the spectral properties of the Iwatsuka model $H_{0}(b)$ and 
derive some decay estimates for the band functions and the corresponding (generalized) eigenfunctions of $H_{0}(b)$. Proofs of Theorem 1.1 for $\lambda$ in general gaps, Theorem 1.4 and Theorem 1.5 are given in Sec. V.

\section{PRELIMINARIES}

\section{A. Variational principle}

In this section we recall some results concerning the variational principle used mainly in Sec. III. All the results are well known, so we omit proofs.

For any sesquilinear form, which is referred to as form in the sequel, $q$ on a Hilbert space, we denote its form domain by $D(q)$. For any semibounded, closable form $q$, there exists a unique self-adjoint operator $A_{q}$ such that the operator domain $D\left(A_{q}\right)$ is a form core for the form closure $\bar{q}$ and the relation $q[u]=\left(A_{q} u, u\right)$ holds for any $u \in D\left(A_{q}\right)$. Throughout the paper, we identify such a form $q$ with the corresponding self-adjoint operator $A_{q}$, and we denote the counting function $N\left(A_{q}<\lambda\right)$ simply by $N(q<\lambda)$ for any real number $\lambda$.

The following result is a consequence of the min-max principle [see, e.g., Reed and Simon (1978, Vol. IV), Colin de Verdiere (1986, Lemma 5.1)].

Lemma 2.1: Let $\left(\mathcal{H}_{j}, q_{j}, D\left(q_{j}\right)\right)$ be a triplet of a Hilbert space $\mathcal{H}_{j}$, a semibounded, closable form $q_{j}$ and the form domain $D\left(q_{j}\right)$ for $j=1,2$ and let $J$ be an isometry from $D\left(q_{1}\right)$ to $D\left(q_{2}\right)$ with respect to norms of $\mathcal{H}_{1}$ and $\mathcal{H}_{2}$, respectively. Suppose that there exist positive constants $C_{1}$ and $C_{2}$ such that $q_{1}[u] \geqslant C_{1} q_{2}[\mathrm{Ju}]-C_{2}\|u\|_{\mathcal{H}_{1}}^{2}$ holds for all $u \in D\left(q_{1}\right)$. Then we have $N\left(q_{1}<\lambda\right) \leqslant N\left(q_{2}\right.$ $\left.<\left(\lambda+C_{2}\right) / C_{1}\right)$ for any $\lambda \in \mathbf{R}$.

For any $\tilde{b} \in C^{1}\left(\mathbf{R}^{2}, \mathbf{R}\right)$, we define

$$
H_{g}(\tilde{b})=-\frac{\partial^{2}}{\partial x^{2}}+\left(\frac{1}{\sqrt{-1}} \frac{\partial}{\partial y}-\tilde{b}(x, y)\right)^{2}-g V(x, y) .
$$

In what follows, for any open subset $\Omega$ in $\mathbf{R}^{2}$, we denote by $\left.H_{g}(\widetilde{b})\right|_{\Omega}$ the minimal self-adjoint realization of $H_{g}(\widetilde{b})$ starting from $C_{0}^{\infty}(\Omega)$, i.e., the Dirichlét realization of $H_{g}(\widetilde{b})$ on $\Omega$.

Proposition 2.2: [Colin de Verdiere (1986, Theorem 1.3)] Let $r$ be a positive number and let $\Lambda$ be a real number. Then we have the upper bound

$$
N\left((-\infty, \Lambda)\left|H_{0}\left(B_{0} x\right)\right|_{Q(0, r)}\right) \leqslant \frac{B_{0}}{2 \pi} r^{2} \#\left\{l \in \mathbf{N} \mid(2 l+1) B_{0}<\Lambda\right\},
$$

where $H_{0}\left(B_{0} x\right)$ is the operator of the form (2.1) with $\widetilde{b}(x, y)=B_{0} x$ and $g=0$, and the lower bound

$$
N\left((-\infty, \Lambda)\left|H_{0}\left(B_{0} x\right)\right|_{Q(0, r)}\right) \geqslant \frac{B_{0}}{2 \pi}\left(r-r_{1}\right)^{2} \#\left\{l \in \mathbf{N} \mid(2 l+1) B_{0}<\Lambda-C r_{1}^{-2}\right\} .
$$

Here, the constant $C$ is independent of $\Lambda, r$ and $r_{1}$ with $0<r_{1}<r$.

The following result is the so-called IMS (Ismagilov, Morgan, Sigal, Simon) localization formula for the magnetic Schrödinger operators [see Cycon et al.].

Lemma 2.3: Let $\widetilde{\Omega}$ be an open subset of $\mathbf{R}^{2}$ and let $\left\{\Omega_{j}\right\}_{j \in J}$ be a locally finite open covering of $\widetilde{\Omega}$. Let $\left\{\chi_{j}\right\}_{j \in J}$ be a partition of unity subject to the covering $\left\{\Omega_{j}\right\}$ satisfying the conditions $\operatorname{supp}\left(\chi_{j}\right) \subset \Omega_{j}, 0 \leqslant \chi_{j} \leqslant 1$ and $\chi_{j} \in C^{1}\left(\Omega_{j}\right)$ for any $j \in J$. Moreover, $\Sigma_{j \in J} \chi_{j}^{2}=1$ on $\widetilde{\Omega}$. Assume that $\tilde{b} \in C^{\infty}(\tilde{\Omega}, \mathbf{R})$. Then we have $\left(H_{g}(\tilde{b}) u, u\right)=\Sigma_{j \in J}\left(H_{g}(\tilde{b}) \chi_{j} u, \chi_{j} u\right)-\left(\Sigma_{l \in J}|\nabla \chi l|^{2} u, u\right)$ for any $u$ $\in C_{0}^{\infty}(\widetilde{\Omega})$.

The next result follows from an elementary inequality $2 X Y \leqslant \varepsilon X^{2}+Y^{2} / \varepsilon$.

Lemma 2.4: Assume that $\tilde{b} \in C^{1}\left(\mathbf{R}^{2}, \mathbf{R}\right)$ and $0<\varepsilon<1$. Then we have 


$$
\begin{gathered}
(1-\varepsilon)\left(H_{0}(\widetilde{b}) u, u\right)-g(V u, u)-(1+1 / \varepsilon)\|(b-\widetilde{b}) u\|^{2} \leqslant\left(H_{g}(b) u, u\right) \\
\quad \leqslant(1+\varepsilon)\left(H_{0}(\widetilde{b}) u, u\right)-g(V u, u)+(1+1 / \varepsilon)\|(b-\widetilde{b}) u\|^{2}
\end{gathered}
$$

for any $u \in C_{0}^{\infty}\left(\mathbf{R}^{2}\right)$.

\section{B. Pseudodifferential operators}

In this section we introduce a class of pseudodifferential operators ( $\Psi$ DOs) and recall some basic results. All the results are well known in the theory of $\Psi$ DOs, so we omit proofs.

For any $m \in \mathbf{R}$ and $a \in C^{\infty}\left(\mathbf{R}^{2}\right)$, we say that $a$ belongs to the class of symbols $S^{m}$ if the quantity

$$
\eta_{\alpha \beta}^{(m)}(a)=\sup _{(x, \xi) \in \mathbf{R}^{2}}\langle x ; \xi\rangle^{-m+\alpha}\left|\partial_{x}^{\beta} \partial_{\xi}^{\alpha} a(x, \xi)\right|
$$

is finite for each $\alpha, \beta \in \mathbf{N}$. The seminorms $\left\{\eta_{\alpha \beta}^{(m)}\right\}_{\alpha, \beta}$ gives a Fréchet space topology on the space $S^{m}$. We set $S^{-\infty}=\cap_{m \in \mathbf{R}} S^{m}$, which coincides with $S(\mathbf{R})$.

The symbol class $S^{m}$ is an example of the class introduced by Beals $(1975)[\Phi(x, \xi)$ $=\langle x ; \xi\rangle, \varphi\langle x, \xi\rangle=1, \lambda=\log \left(\langle x ; \xi\rangle^{m}\right)$ and $S_{\Phi, \varphi}^{\lambda}=S^{m}$ in his convention], or by Robert (1978), Dauge and Robert (1987) $\left[m=\langle x ; \xi\rangle^{m}, \phi=\langle x ; \xi\rangle, \varphi=1, S(m ; \phi, \varphi)=S^{m}\right.$ in their convention]. Hence, by the standard argument as in Beals (1975) or in Hörmander (1979) $\left[g_{x, \xi}(y, \eta)=|y|^{2}+|\eta|^{2} /\langle x ; \xi\rangle^{2}\right.$ in his convention], one can find that, for any $a \in S^{m}$, the associated $\Psi \mathrm{DO}$,

$$
O p(a) u(x)=\iint_{\mathbf{R}^{2}} e^{\sqrt{-1}(x-y) \xi} a\left(\frac{x+y}{2}, \xi\right) u(y) \mathrm{d} y \mathrm{~d} \xi
$$

is a well-defined oscillatory integral for any $u \in S(\mathbf{R})$. Here, we set $\mathrm{d} \xi=\mathrm{d} \xi /(2 \pi)$. Moreover, $O p(a)$ maps $\mathcal{S}(\mathbf{R})$ to itself continuously [so, extends to a continuous map from $\mathcal{S}^{\prime}(\mathbf{R})$ to itself by duality based on $L^{2}$-norm]. For an operator $A$ from $\mathcal{S}^{\prime}(\mathbf{R})$ to $\mathcal{S}^{\prime}(\mathbf{R})$, we say $A \in O p S^{m}$ if $A$ is expressed as $O p(a)$ for some $a \in S^{m}$.

Note that the original results in Beals (1975) are formulated in terms of the standard quantization $\iint_{\mathbf{R}^{2} e^{\sqrt{-1}(x-y) \xi}} a(x, \xi) u(y) \mathrm{d} y \mathrm{~d} \xi$. However, all the corresponding results below are still valid for the Weyl quantization; to see this, it suffices to chase the proofs in Beals (1975) carefully, or use the relation between the standard and the Weyl quantizations as in Theorem 4.5 in Hörmander (1979). For omitted proofs, we refer to Proposition 6.17, Theorems 6.1, 7.2, and 7.7 in Beals (1975), and for Lemma 2.7 below we refer to Proposition 26.2 in Shubin (1987). Although the class of symbols considered in Shubin's book is slightly defferent from the class $S^{m}$ above, the proof of Proposition 26.2 in his book is valid also for symbols in $S^{m}$ with obvious modifications.

Lemma 2.5: Let $m, m^{\prime} \in \mathbf{R}$. We have the following assertions.

(1) If $A \in O p S^{m}$ and $B \in O p S^{m^{\prime}}$, then $A B \in O p S^{m+m^{\prime}}$ and the symbol of $A B$ has an asymptotic expansion as usual.

(2) If $A \in O p S^{m}$, then $A^{*} \in O p S^{m}$ and the symbol of $A^{*}$ is expressed as usual.

(3) If $A \in O p S^{0}$, then $A$ defines a bounded operator on $L^{2}(\mathbf{R})$. Moreover, if $A \in O p S^{m}$ for some negative $m$, then A defines a compact operator on $L^{2}(\mathbf{R})$.

(4) If $m>0$ and $a \in S^{-m}$, then there exists $l \in \mathbf{N}$ such that $\|O p(a)\|_{\mathbf{B}\left(L^{2}(\mathbf{R})\right)} \leqslant C \Sigma_{\alpha+\beta \leqslant l} \eta_{\alpha \beta}^{(-m)}(a)$ holds for some constant $C>0$, independent of $a$.

(5) If $A \in O p S^{-\infty}$, then $A$ maps $\mathcal{S}^{\prime}(\mathbf{R})$ to $\mathcal{S}(\mathbf{R})$ continuously.

We introduce the weighted Sobolev space $H^{m}$ as the (finite) linear hull of the set $\{A u \mid u$ $\left.\in L^{2}(\mathbf{R}), A \in O p S^{-m}\right\}$ equipped with the weakest topology which makes the map $\left(A: L^{2}(\mathbf{R})\right.$ $\left.\rightarrow H^{m}\right)$ continuous for all $A \in O p S^{-m}$. The basic properties of $H^{m}$ are summarized as follows.

Lemma 2.6:

(1) The space $H^{0}$ coincides with $L^{2}(\mathbf{R})$ topologically.

(2) The embeddings $\mathcal{S}(\mathbf{R}) \subset H^{m} \subset \mathcal{S}^{\prime}(\mathbf{R})$ are densely and continuously for any $m$. 
(3) $H^{m} \subset H^{m^{\prime}}$ holds if $m^{\prime}<m$, and $\left(H^{m}\right)^{\prime}=H^{-m}$ topologically for any $m$.

(4) If $A \in O p S^{m}$, then A maps $H^{m+m^{\prime}}$ to $H^{m^{\prime}}$ continuously for all $m, m^{\prime}$.

(5) For any $m, m^{\prime}$, there exists $A \in O p S^{m}$ which gives a topologically isomorphism from $H^{m+m^{\prime}}$ onto $H^{m^{\prime}}$. In particular, $H^{m}$ has the topology of a Hilbert space for any $m$.

Lemma 2.7: Let $D_{0}$ be a dense subspace of $\mathcal{S}(\mathbf{R})$. Let $m>0$ and $a \in S^{m}$. Assume that $O p(a)$ is symmetric on $D_{0}$ and $|a(x, \xi)| \geqslant C\langle x ; \xi\rangle^{m}$ holds for some $C>0$. Then

(1) The operator $\left.O p(a)\right|_{D_{0}}$ [the restriction of $O p(a)$ on $\left.D_{0}\right]$ is essentially self-adjoint, and $D\left(\overline{\left.O p(a)\right|_{D_{0}}}\right)$ coincides with $\left\{u \in L^{2}(\mathbf{R}) \mid O p(a) u \in L^{2}(\mathbf{R})\right\}$.

(2) The space $D\left(\overline{\left.O p(a)\right|_{D_{0}}}\right)$ equipped with graph norm coincides with $H^{m}$ topologically.

The following result concerning the eigenvalue asymptotics of $\Psi$ DOs of negative order plays an important role in Sec. V.

Proposition 2.8: [Dauge and Robert (1987, Theorem 1.3)] Let $m>0$ and let $a \in S^{-m}$ and $a$ be real valued. Assume that the following two estimates,

$$
\lim _{\varepsilon \downarrow 0} \limsup _{\mu \downarrow 0} \mu^{2 / m} \operatorname{Vol}\left\{(x, \xi) \in \mathbf{R}^{2} \mid(1-\varepsilon) \mu< \pm a(x, \xi)<(1+\varepsilon) \mu\right\}=0,
$$

are satisfied. Then we have $N( \pm O p(a)>\mu)=(1 / 2 \pi) \operatorname{Vol}\left\{(z, \xi) \in \mathbf{R}^{2} \mid \pm a(x, \xi)>\mu\right\}+o\left(\mu^{-2 / m}\right)$ as $\mu \downarrow 0$, respectively.

Note that the conditions (2.3) imply the condition $(T)$ in Dauge and Robert (1987) for the volume functions $\operatorname{Vol}\{z \mid \pm a(z)>\mu\}$. As an immediate consequence of Proposition 2.8, for any $m>0$ and any $a \in S^{-m}$, the following rough estimate,

$$
N( \pm O p(a)>\mu)=O\left(\mu^{-2 / m}\right),
$$

holds as $\mu \downarrow 0$.

\section{PROOF OF THEOREM 1.1 IN THE CASE OF $\lambda<B_{-}$}

\section{A. Upper bound for $N\left(H_{g}(b)<\lambda\right)$}

\section{Partition of $R^{2}$ and sesquilinear forms}

In this section, we give a proof of Theorem 1.1 in the case of $\lambda<\Lambda_{0}^{-}\left(=B_{-}\right)$. For general $\lambda$ $>\Lambda_{0}^{+}$the proof of Theorem 1.1 given in Sec. V needs this special case, as in Raikov (1993).

We introduce a partition of $\mathbf{R}^{2}$ and a corresponding partition of unity. Let $K=\{0,1,+,-\}$. Take and fix positive numbers $\alpha, \beta$, and $\sigma$ so that

$$
0<\sigma<\min \left\{\frac{1}{4 m}, \frac{1}{2-m}, \frac{M}{m(1+M)}, \frac{1}{2 m(m+1)}\right\}, \quad \alpha=\frac{1}{m}-2 \sigma, \quad \beta=\frac{1}{m}+\sigma .
$$

Note that $0<\sigma<\alpha<1 / m<\beta$. For any $g>0$, we set $\Omega_{0}=\left\{(x, y) \in \mathbf{R}^{2}|| x\left|\leqslant g^{\alpha},\right| y \mid \leqslant g^{\beta}\right\}, \Omega_{ \pm}$ $=\left\{(x, y) \in \mathbf{R}^{2}\left|g^{\alpha} \leqslant \pm x \leqslant g^{\beta},\right| y \mid \leqslant g^{\beta}\right\}, \quad$ respectively, $\quad \Omega_{1}=\mathbf{R}^{2} \backslash\left(\Omega_{0} \cup \Omega_{+} \cup \Omega_{-}\right) \quad$ and $\quad \widetilde{\Omega}_{k}=\{z$ $\left.\in \mathbf{R}^{2} \mid \operatorname{dist}\left(z, \Omega_{k}\right)<g^{\sigma}\right\}$ for any $k \in K$. Let $\left\{\varphi_{k}\right\}_{k \in K}$ be a partition of unity subject to the open covering $\left\{\widetilde{\Omega}_{k}\right\}_{k \in K}$ of $\mathbf{R}^{2}$ satisfying the following:

(P.1) $\varphi_{k} \in C^{\infty}\left(\widetilde{\Omega}_{k}\right), \operatorname{supp}\left(\varphi_{k}\right) \subset \widetilde{\Omega}_{k}$ and $0 \leqslant \varphi_{k} \leqslant 1$ hold, and for each multi-index $\gamma$, there exists $C_{\gamma}>0$ such that $\sup _{z \in \mathbf{R}^{2}}\left|\varphi_{k}(z)\right| \leqslant C_{\gamma} g^{-|\gamma| \sigma}$ holds for any $k \in K$. Moreover, $\Sigma_{k \in K} \varphi_{k}^{2}=1$ holds on $\mathbf{R}^{2}$.

For each $k \in K$, we define a form $q_{k}$ by $q_{k}[u]=\left(H_{g}(b) u, u\right)-\left(\Sigma_{l \in K}\left|\nabla_{\varphi l}\right|^{2} u, u\right)$ with form domain $C_{0}^{\infty}\left(\widetilde{\Omega}_{k}\right)$. Then it follows from Lemma 2.3 that $\left(H_{g}(b) u, u\right)=\Sigma_{k \in K} q_{k}\left[\varphi_{k} u\right]$ holds for any $u$ $\in C_{0}^{\infty}\left(\mathbf{R}^{2}\right)$. Considering the isometry $J$ from $L^{2}\left(\mathbf{R}^{2}\right)$ to $\oplus_{k \in K} L^{2}\left(\widetilde{\Omega}_{k}\right)$ defined by $J(u)=\oplus_{k \in K}\left(\varphi_{k} u\right)$, by Lemma 2.1, we find that $N\left(H_{g}(b)<\lambda\right) \leqslant \Sigma_{k \in K} N\left(q_{k}<\lambda\right)$ holds for any $g>0$. 


\section{Estimates of $N\left(q_{0}<\lambda\right)$ and $N\left(q_{1}<\lambda\right)$}

In what follows we use the symbols $c$ and $C$ (possibly with superscripts or subscripts) to denote various positive constants in estimates, which may vary from line to line.

Let $0<\eta<1$ and let $J=\left\{j \in \mathbf{Z}^{2} \mid \widetilde{\Omega}_{0} \cap Q(j, 1) \neq \varnothing\right\}$. Let $\left\{\chi_{j}\right\}_{j \in J}$ be a partition of unity subject to the open covering $\{Q(j, 1+\eta)\}_{j \in J}$ of $\widetilde{\Omega}_{0}$ satisfying the following:

$(P .2) \chi_{j} \in C_{0}^{\infty}(Q(j, 1+\eta))$, and $0 \leqslant \chi_{j} \leqslant 1$ hold, and for each multi-index $\gamma$, there exists $C_{\gamma}$ $>0$, independent of $j, \eta$, such that $\sup _{z \in \mathbf{R}^{2}}\left|\chi_{j}(z)\right| \leqslant C_{\gamma} \eta^{-|\gamma|}$ holds for any $j \in J$. Moreover, $\Sigma_{j \in J} \chi_{j}^{2}=1$ holds on $\widetilde{\Omega}_{0}$.

For each $j=\left(j_{1}, j_{2}\right) \in J$, we introduce the auxiliary magnetic potential $b_{j}(x)=\int_{0}^{j_{1}} B(t) \mathrm{d} t+B\left(j_{1}\right)$ $\times\left(x-j_{1}\right)$, which gives the constant magnetic field $B\left(j_{1}\right)$.

Lemma 3.1: Let $q_{0}$ and $\lambda$ be as above. We have $N\left(q_{0}<\lambda\right)=o\left(g^{2 / m}\right)$ as $g \rightarrow \infty$.

Proof: By Lemmata 2.3, 2.1, 2.4 and Proposition 2.2, we have the estimate

$$
\begin{aligned}
N\left(q_{0}<\lambda\right) & \leqslant \sum_{j \in J} N\left((1-\varepsilon) H_{0}\left(b_{j}\right)-C g\langle j\rangle^{-m}-C_{\eta \varepsilon}<\lambda\right) \\
& \leqslant \sum_{j=\left(j_{1}, j_{2}\right) \in J} \frac{B\left(j_{1}\right)}{2 \pi}(1+\eta)^{2} \#\left\{l \in \mathbf{N} \mid(1-\varepsilon)(2 l+1) B\left(j_{1}\right)<\lambda+C g\langle j\rangle^{-m}+C_{\eta \varepsilon}\right\} \\
& \leqslant C_{\eta \varepsilon \lambda}(\# J)+C_{\eta \varepsilon \lambda} g \sum_{j \in J}\langle j\rangle^{-m}
\end{aligned}
$$

for any small $\varepsilon>0$, where we used (P.2) and the fact that the estimates $|V(z)| \leqslant C\langle j\rangle^{-m}$ and $\left|b(x)-b_{j}(x)\right| \leqslant\left|\int_{j_{1}}^{x}\left(B(t)-B\left(j_{1}\right)\right) \mathrm{d} t\right| \leqslant 2(1+\eta) B_{+}$hold on $Q(j, 1+\eta)$ in the first inequality. By the definition of $J$, there exists $C>0$, independent of $g$, such that $\sharp(J) \leqslant C \operatorname{Vol}\left(\widetilde{\Omega}_{0}\right) \leqslant C g^{\alpha+\beta}$ $=o\left(g^{2 / m}\right)$ as $g \rightarrow \infty$, since $\alpha+\beta=2 / m-\sigma$. The second term on the right-hand side (rhs) of (3.1) is less than or equal to

$$
C g \int_{\tilde{\Omega}_{0}}\langle z\rangle^{-m} \mathrm{~d} z \leqslant 4 C g \int_{0}^{g^{\alpha}+g^{\sigma}} \mathrm{d} x \int_{0}^{g^{\beta}+g^{\sigma}} \mathrm{d} y\langle x ; y\rangle^{-m} .
$$

To estimate the above integral, we use the following elementary estimate: If $m>0$ and $1<A$ $<B$, then there exists $C_{m}>0$, independent of $A, B$, such that

$$
\int_{0}^{A} \mathrm{~d} x \int_{0}^{B} \mathrm{~d} y\langle x ; y\rangle^{-m} \leqslant\left\{\begin{array}{l}
C_{m} A B^{1-m} \quad \text { if } 0<m<1, \\
C_{1} A \log B \quad \text { if } m=1, \\
C_{m}\left(A B^{1-m}+1\right) \quad \text { if } m>1 .
\end{array}\right.
$$

Then, if $0<m<1$, the rhs of (3.2) is estimated from above by $C_{m} g\left(g^{\alpha}+g^{\sigma}\right)\left(g^{\beta}+g^{\sigma}\right)^{1-m}$ $=o\left(g^{1+\alpha+\beta(1-m)}\right)=o\left(g^{2 / m}\right)$ as $g \rightarrow \infty$. Similarly, if $m=1$, the rhs of (3.2) is estimated from above by $C_{1} g\left(g^{\alpha}+g^{\sigma}\right) \log \left(g^{\beta}+g^{\sigma}\right)=o\left(g^{1+\alpha} \log g\right)=o\left(g^{2 / m}\right)$ as $g \rightarrow \infty$, and if $1<m<2$, the rhs of (3.2) is estimated from above by $C_{m} g\left(\left(g^{\alpha}+g^{\sigma}\right)\left(g^{\beta}+g^{\sigma}\right)^{1-m}+1\right)=o\left(g^{1+\alpha-\beta(m-1)}\right)=o\left(g^{2 / m}\right)$ as $g \rightarrow \infty$. This completes the proof.

Lemma 3.2: Let $q_{1}$ and $\lambda$ be as above. We have $N\left(q_{1}<\lambda\right)=0$ for large $g>0$.

Proof: For any $u \in C_{0}^{\infty}\left(\widetilde{\Omega}_{1}\right)$, we have $q_{1}[u]=\left(H_{g}(b) u, u\right)-g(V u, u)-\left(\Sigma_{l \in K}\left|\nabla \varphi_{l}\right|^{2} u, u\right)$ $\geqslant\left(H_{g}(b) u, u\right)-C\left(g^{1-m \beta}+g^{-2 \sigma}\right)\|u\|^{2}=\left(H_{g}(b) u, u\right)-o(1)\|u\|^{2}$ as $g \rightarrow \infty$, where we used $(V .1),(P .1)$ and the fact that $1-m \beta=1-m(1 / m+\sigma)=-m \sigma<0$. Then the min-max argument yields that $N\left(q_{1}<\lambda\right) \leqslant N\left(\left.H_{0}(b)\right|_{\Omega_{1}}<\lambda+o(1)\right)$ holds as $g \rightarrow \infty$. Hence we conclude that $N\left(\left.H_{0}(b)\right|_{\tilde{\Omega}_{1}}\right.$ $<\lambda+o(1))=0 \quad$ for large $g$ since $\inf \operatorname{Spec}\left(\left.H_{0}(b)\right|_{\Omega_{1}}\right)=\inf _{u \in C_{0}^{\infty}\left(\tilde{\Omega}_{1}\right),\|u\|=1}\left(H_{0}(b) u, u\right) \geqslant \inf$ $\operatorname{Spec}\left(H_{0}(b)\right)\left(=B_{-}>\lambda\right)$ holds as $g \rightarrow \infty$ again by the min-max argument. 


\section{Estimate of $N\left(q_{ \pm}<\lambda\right)$}

Let $0<\eta<1$. For any $j=\left(j_{1}, j_{1}\right) \in \mathbf{Z}^{2}$, we set

$$
\begin{gathered}
Q_{j}=\left\{(x, y) \in \mathbf{R}^{2} \mid j_{1}<g^{-\sigma} x<j_{1}+1, j_{2}<g^{-\sigma} y<j_{2}+1\right\}, \\
Q_{j \eta}=\left\{z \in \mathbf{R}^{2} \mid \operatorname{dist}\left(z, Q_{j}\right)<\eta g^{\sigma}\right\}
\end{gathered}
$$

and $J_{ \pm}=\left\{j \in \mathbf{Z}^{2} \mid Q_{j} \cap \widetilde{\Omega}_{ \pm} \neq \varnothing\right\}$. Let $\left\{\chi_{j}\right\}_{j \in J_{ \pm}}$be a partition of unity subject to the covering $\left\{Q_{j \eta}\right\}_{j \in J_{ \pm}}$ of $\widetilde{\Omega}_{ \pm}$satisfying the following conditions (respectively, for \pm ):

$(P .3)_{ \pm} \chi_{j} \in C_{0}^{\infty}\left(Q_{j \eta}\right), 0 \leqslant \chi_{j} \leqslant 1$, and for each multi-index $\gamma$, there exists $C_{\gamma}>0$, independent of $g, \eta, j$, such that $\sup _{z \in \mathbf{R}^{2}}\left|\partial^{\gamma} \chi_{j}(z)\right| \leqslant C_{\gamma}\left(\eta g^{\sigma}\right)^{-|\gamma|}$ holds for any $j \in J_{ \pm}$. Moreover, $\Sigma_{j \in J_{ \pm}} \chi_{j}^{2}=1$ holds on $\widetilde{\Omega}_{ \pm}$.

In what follows, for simplicity, we omit the phrase "respectively (for \pm )" if there is no fear of confusion.

Let $z_{j}=\left(x_{j}, y_{j}\right)$ be the center of $Q_{j}$ and we introduce the auxiliary magnetic potentials,

$$
b_{j, \pm}(x)=\int_{0}^{x_{j}} B(t) \mathrm{d} t+B_{ \pm}\left(x-x_{j}\right)
$$

which gives the constant magnetic field $B_{ \pm}$.

Lemma 3.3: Let $j \in J_{ \pm}$. There exists $C>0$, independent of $j, g$, and $\eta$, such that $\left\|\left(b-b_{j, \pm}\right) u\right\|$ $\leqslant C g^{\sigma-M \alpha}\|u\|$ holds for any $u \in C_{0}^{\infty}\left(Q_{j \eta}\right)$, and for any $g \geqslant 1$.

Proof: On $\quad Q_{j \eta}, \quad$ we have $\left|b(x)-b_{j, \pm}(x)\right| \leqslant\left|\int_{x_{j}}^{x}\left(B(t)-B_{ \pm}\right) \mathrm{d} t\right| \leqslant C\left\langle g^{\alpha}-g^{\sigma}-\eta g^{\sigma}\right\rangle^{-M}\left|x-x_{j}\right|$ $\leqslant C g^{-M \alpha+\sigma}$ as $g \rightarrow \infty$, where we used (B.2) in the second inequality.

Lemma 3.4: Let $j \in J_{ \pm}$. There exists $C>0$, independent of $j, g$, and $\eta$ such that (1 $\left.-C g^{-(\alpha-2 \sigma)}\right) V(z) \leqslant V\left(z^{\prime}\right) \leqslant\left(1+C g^{-(\alpha-2 \sigma)}\right) V(z)$ holds for any $z, z^{\prime} \in Q_{j \eta}$. Here, $\bar{Q}$ stands for the closure of $Q$.

Proof: Let $z, z^{\prime} \in \overline{Q_{j \eta}}$. If we write $z=(x, y)$, then

$$
|z| \geqslant|x| \geqslant g^{\alpha}-g^{\sigma}-(1+\eta) g^{\sigma} \geqslant g^{\alpha} / 2
$$

and $|z| \leqslant\left|z^{\prime}\right|+\left|z-z^{\prime}\right| \leqslant\left|z^{\prime}\right|+\sqrt{2}(1+\eta) g^{\sigma} \leqslant\left|z^{\prime}\right|+C g^{-(\alpha-\sigma)}|z|$ hold for large $g>0$, so there exists $C$ $>0$, independent of $j, g, z, z^{\prime}$, such that

$$
C^{-1}\langle z\rangle \leqslant\left\langle z^{\prime}\right\rangle \leqslant C\langle z\rangle
$$

holds. The first order Taylor expansion yields $\left|V(z)-V\left(z^{\prime}\right)\right| \leqslant\left|z-z^{\prime}\right| \sup _{w \in Q_{j \eta}}|\nabla V(w)| \leqslant C(1$ $+\eta)^{2} g^{2 \sigma} \sup _{w \in Q_{j \eta}}\langle w\rangle^{-m-1} \leqslant C(1+\eta)^{2} g^{-(\alpha-2 \sigma)} \sup _{w \in Q_{j \eta}}\langle w\rangle^{-m} \leqslant C g^{-(\alpha-2 \sigma)} V(z)$ for large $g$, where we used (3.6) in the third inequality, (V.1), (V.2) in the second and the fourth inequalities, (3.7) in the last inequality. Here, the constant $C$ is independent of $j, \eta, g, z$, and $z^{\prime}$. This proves the lemma

Lemma 3.5: For any $\varepsilon$ satisfying $0<\varepsilon<1$, we have

$$
N\left(q_{ \pm}<\lambda\right) \leqslant(1+\varepsilon)^{2} \frac{B_{ \pm}}{2 \pi} \sum_{l \in N} \operatorname{Vol}\left(\Omega_{ \pm}^{(+)}(\varepsilon, l)\right),
$$

where we set $\Omega_{ \pm}^{(+)}(\varepsilon, l)=\left\{z \in \mathbf{R}^{2} \mid \pm x \geqslant 0,(1-2 \varepsilon) \Lambda_{l}^{ \pm}<\lambda+g(1+\varepsilon) V(z)\right\}$. In fact, the sum on the rhs of (3.8) terminates for each $\varepsilon$ and $g$.

Proof: By Lemma 2.1 and (P.3), there exists $C>0$, independent of $j, g, \eta$, such that 


$$
\begin{aligned}
q_{ \pm}[u] \geqslant & \sum_{j \in J_{ \pm}}\left((1-\varepsilon)\left(H_{0}\left(b_{j, \pm}\right) \chi_{j} u, \chi_{j} u\right)-g\left(V \chi_{j} u, \chi_{j} u\right)-(1+1 / \varepsilon)\left\|\left(b-b_{j, \pm}\right) \chi_{j} u\right\|^{2}-C\left(\eta g^{\sigma}\right)^{-2}\left\|\chi_{j} u\right\|^{2}\right. \\
& \left.-C g^{-2 \sigma}\left\|\chi_{j} u\right\|^{2}\right) \geqslant \sum_{j \in J_{ \pm}}\left((1-\varepsilon)\left(H_{0}\left(b_{j, \pm}\right) \chi_{j} u, \chi_{j} u\right)-g \sup _{Q_{j \eta}}|V|\left(\chi_{j} u, \chi_{j} u\right)-o(1)\left\|\chi_{j} u\right\|^{2}\right)
\end{aligned}
$$

holds for any $u \in C_{0}^{\infty}\left(\widetilde{\Omega}_{ \pm}\right)$, where we write $\sup _{Q}|V|$ for $\sup _{z \in Q}|V(z)|$ for short. Then the min-max theorem yields that

$$
\begin{aligned}
N\left(q_{ \pm}\right. & <\lambda) \leqslant \sum_{j \in J_{ \pm}} N\left((1-2 \varepsilon) H_{0}\left(b_{j, \pm}\right)<\lambda+g \sup _{Q_{j \eta}}|V|\right) \\
& \leqslant(1+\eta)^{2} g^{2 \sigma} \frac{B_{ \pm}}{2 \pi} \sum_{j \in J_{ \pm}} \#\left\{l \in \mathbf{N}\left|(1-2 \varepsilon) \Lambda_{l}^{ \pm}<\lambda+g \sup _{Q_{j \eta}}\right| V \mid\right\} \\
& =(1+\eta)^{2} g^{2 \sigma} \frac{B_{ \pm}}{2 \pi} \sum_{l \in N} \sum_{j \in J_{ \pm}} F\left((1-2 \varepsilon) \Lambda_{l}^{ \pm}<\lambda+g \sup _{Q_{j \eta}}|V|\right)
\end{aligned}
$$

holds for large $g$, where we used Proposition 2.2 in the second inequality. Here, $F(P)=0$ if $P$ is true and $F(P)=1$ if $P$ is false.

Set $\widetilde{J}_{ \pm}=\left\{j \in J_{ \pm} \mid Q_{j} \subset \Omega_{ \pm}^{(+)}(\varepsilon, l)\right\}$. We claim that, if $j \in J_{ \pm} \backslash \widetilde{J}_{ \pm}$, then $F\left((1-2 \varepsilon) \Lambda_{l}^{ \pm}<\lambda\right.$ $\left.+g \sup _{Q_{j \eta}}|V|\right)=0$ holds for large $g>0$ uniformly in $j, l$. Indeed, for such $j$, there exists $\bar{z} \in Q_{j}$ such that $\bar{z} \notin \Omega_{ \pm}^{(+)}(\varepsilon, l)$, i.e.,

$$
(1-2 \varepsilon) \Lambda_{l}^{ \pm} \geqslant \lambda+g(1+\varepsilon) V(\bar{z}) .
$$

Note that if we write $\bar{z}=(\bar{x}, \bar{y}) \in Q_{j}$, then $\pm \bar{x} \geqslant 0$ since $0<\sigma<\alpha$. Then we find that, for any $\varepsilon$ $>0 \quad$ fixed, $\quad(1-2 \varepsilon) \Lambda_{l}^{ \pm}-\lambda-g \sup _{Q_{j \eta}}|V|=(1-2 \varepsilon) \Lambda_{l}^{ \pm}-\lambda-g(1+\varepsilon) V(\bar{z})+g\left((1+\varepsilon) V(\bar{z})-\sup _{Q_{j \eta}}|V|\right)$ $\geqslant g\left((1+\varepsilon) V(\bar{z})-\sup _{Q_{j \eta}}|V|\right) \geqslant g\left((1+\varepsilon) V(\bar{z})-\left(1+C g^{-(\alpha-2 \sigma)}\right) V(\bar{z})\right)=g\left(\varepsilon-C g^{-(\alpha-2 \alpha)}\right) V(\bar{z}) \geqslant 0 \quad$ holds for large $g$ (uniformly in $j, l$ ), where we used (3.10) in the first inequality, Lemma 3.4 in the second inequality and $(V .2)$ in the last. This shows the claim.

Hence, it follows that, for large $g$, the rhs of (3.9) is less than or equal to (1 $+\eta)^{2}\left(B_{ \pm} / 2 \pi\right) \Sigma_{l \in N} \Sigma_{j \in \bar{J}_{ \pm}} \operatorname{Vol}\left(Q_{j}\right) \leqslant(1+\eta)^{2}(B \pm / 2 \pi) \Sigma_{l \in N} \operatorname{Vol}\left(\Omega_{ \pm}^{(+)}(\varepsilon, l)\right)$. Then the lemma follows since the set $\Omega_{ \pm}^{(+)}$is empty if $l$ is so large that $(1-2 \varepsilon) \Lambda_{l}^{ \pm} \geqslant \lambda+C g \sup _{\mathbf{R}^{2}}|V|$.

Lemma 3.6: Assume that $\lambda<B_{-}$. Then we have $\lim _{\sup } \rightarrow \infty g^{-2 / m} N_{g}^{+}(\lambda) \leqslant \Sigma_{l \in \mathbf{N}} \nu_{l}(\lambda)$.

Proof: Let $\Omega_{ \pm}^{(+)}(\varepsilon, l)$ be as in the previous lemma. Set $I_{0}=\operatorname{Vol}\left\{z \in \Omega_{ \pm}^{(+)}(\varepsilon, l)|| z \mid \leqslant R\right\}$ and $I_{\infty}^{ \pm}$ $=\left\{z=(x, y) \in \Omega_{ \pm}^{(+)}(\varepsilon, l)| \pm x \geqslant 0| z \mid, \geqslant R\right\}$. Then $\operatorname{Vol}\left(\Omega_{ \pm}^{(+)}(\varepsilon, l)\right)=I_{0}+I_{\infty}^{+}+I_{\infty}^{-}$. We observe that $I_{0}$

$\leqslant \pi R^{2}$, and $I_{\infty}^{ \pm} \leqslant \frac{1}{2} g^{2 / m}\left(\Lambda_{l}^{ \pm}-\lambda\right)^{-2 / m} \int_{S_{ \pm}^{1}} v(\omega)^{2 / m} \mathrm{~d} \omega+O(1)$ as $\varepsilon \downarrow 0$ (the remainder term is uniformly bounded in $g$ ), since by (V.3), for any small $\varepsilon>0$, there exists $R>0$ such that $\left|r^{m} V(z)-v(\omega)\right|$ $<\varepsilon$ if $r=|z| \geqslant R$. Then, taking a limit $\varepsilon \downarrow 0$ in (3.8), we derive from Lemmas 3.2, 3.1, and 3.5 that $\lim \sup _{g \rightarrow \infty} g^{-2 / m} N\left(H_{g}(b)<\lambda\right) \leqslant \Sigma_{l \in N} \nu_{l}(\lambda)$, by Lebesgue's dominated convergence theorem. The lemma follows since $N\left(H_{g}(b)<\lambda\right)=N_{g}^{+}(\lambda)$ holds if $\lambda<B_{-}$because of the non-negativity of $V$.

\section{B. Lower bound for $N\left(H_{g}(b)<\lambda\right)$}

Let $K$ and $\left\{\Omega_{k}\right\}_{k \in K}$ be as in the beginning of this section. Applying Lemma 2.1 to the pair of triplets $\left(\Sigma_{k \in K} \oplus L^{2}\left(\Omega_{k}\right), t_{1}, \oplus_{k \in K} C_{0}^{\infty}\left(\Omega_{k}\right)\right)$ and $\left(L^{2}\left(\mathbf{R}^{2}\right), t_{2}, C_{0}^{\infty}\left(\mathbf{R}^{2}\right)\right)$, where we set $t_{1}\left[\oplus_{k \in K} v_{k}\right]$ $=\Sigma_{k \in K}\left(\left.H_{g}(b)\right|_{\Omega_{k}} v_{k}, v_{k}\right)$ for $\oplus_{k \in K}\left(v_{k}\right) \in \oplus_{k \in K} C_{0}^{\infty}\left(\Omega_{k}\right), t_{2}[u]=\left(H_{g}(b) u, u\right)$ for $u \in C_{0}^{\infty}\left(\mathbf{R}^{2}\right)$ and $J: \oplus_{k \in K} C_{0}^{\infty}\left(\Omega_{k}\right) \ni \oplus_{k \in K}\left(v_{k}\right) \mapsto \Sigma_{k \in K} v_{k} \in C_{0}^{\infty}\left(\mathbf{R}^{2}\right)$, we have

$$
N\left(H_{g}(b)<\lambda\right) \geqslant \sum_{k \in K} N\left(\left.H_{g}(b)\right|_{\Omega_{k}}<\lambda\right) .
$$

As in the proof of Lemma 3.1, we can show that 


$$
N\left(\left.H_{g}(b)\right|_{\Omega_{0}}<\lambda\right)=o\left(g^{2 / m}\right)
$$

as $g \rightarrow \infty$, using the upper estimate in Lemma 2.4. Also, as in the proof of Lemma 3.2, we can show that

$$
N\left(\left.H_{g}(b)\right|_{\Omega_{1}}<\lambda\right) \leqslant N\left(\left.H_{0}(b)\right|_{\Omega_{1}}<\lambda+o(1)\right)=0
$$

holds for large $g>0$.

Lemma 3.7: For any $l \in \mathbf{N}$ and any $\varepsilon$ satisfying $0<\varepsilon<1$, set

$$
\Omega_{ \pm}^{(-)}(\varepsilon, l)=\left\{z \in \mathbf{R}^{2} \mid \pm x \geqslant 0,(1+2 \varepsilon) \Lambda_{l}^{ \pm}<\lambda+(1-\varepsilon) g V(z)\right\},
$$

respectively. Then for any $\varepsilon$ satisfying $0<\varepsilon<1$, we have

$$
N\left(\left.H_{g}(b)\right|_{\Omega_{ \pm}}<\lambda\right) \geqslant(1-\varepsilon)^{2} \frac{B_{ \pm}}{2 \pi} \sum_{l \in \mathrm{N}} \operatorname{Vol}\left(\Omega_{ \pm}^{(-)}(\varepsilon, l)\right)+o\left(g^{2 / m}\right)
$$

as $g \rightarrow \infty$, respectively.

Proof: Let $0<\eta<1$ and $j \in \mathbf{Z}^{2}$. Let $Q_{j}$ and $Q_{j \eta}$ be the cubes defined by (3.3) and (3.4), respectively. Set $J_{ \pm}^{\prime}=\left\{j \in \mathbf{Z}^{2} \mid Q_{j} \subset \widetilde{\Omega}_{ \pm}, Q_{j} \cap \Omega_{ \pm}^{(-)}(\varepsilon, l) \neq \varnothing\right\}$, respectively. Let $b_{j, \pm}$ be as in (3.5). Then, as in the proof of Lemma 3.5, it follows from Lemma 2.4, Lemma 2.1, and Lemma 2.3 that, for any $\varepsilon$ satisfying $0<\varepsilon<1$,

$$
\begin{aligned}
& N\left(\left.H_{g}(b)\right|_{\Omega_{ \pm}}<\lambda\right) \geqslant \sum_{j \in J_{ \pm}^{\prime}} N\left((1+2 \varepsilon) H_{0}(b)\left|Q_{j}<\lambda+g \sup _{Q_{j \eta}}\right| V \mid\right) \\
& \geqslant(1-\varepsilon)^{2} \frac{B_{ \pm}}{2 \pi} \sum_{j \in J_{ \pm}^{\prime}} \operatorname{Vol}\left(Q_{j}\right) \#\left\{l \in \mathbf{N}\left|(1+2 \varepsilon) \Lambda_{l}^{ \pm}<\lambda+g \sup _{Q_{j \eta}}\right| V \mid\right\} \\
& \geqslant(1-\varepsilon)^{2} \frac{B_{ \pm}}{2 \pi} \sum_{l \in \mathrm{N}} \sum_{j \in J_{ \pm}^{\prime}} \operatorname{Vol}\left(Q_{j}\right) F\left((1+2 \varepsilon) \Lambda_{l}^{ \pm}<\lambda+g \sup _{Q_{j \eta}}|V|\right),
\end{aligned}
$$

where we used Proposition 2.2 with $r_{1}=\varepsilon$ in the third inequality.

We claim that, if $j \in J_{ \pm}^{\prime}$, then $F\left((1+2 \varepsilon) \Lambda_{l}^{ \pm}<\lambda+g \sup _{Q_{i \eta}}|V|\right)=1$ holds for large $g>0$ uniformly in $j, l$. Indeed, if $j \in J_{ \pm}^{\prime}$, there exists $\bar{z} \in Q_{j} \cap \Omega_{ \pm}^{(-)}(\varepsilon, l)$, i.e., $\bar{z} \in Q_{j}$ and $(1+2 \varepsilon) \Lambda_{l}^{ \pm}<\lambda+g(1$ $-\varepsilon) V(\bar{z})$ hold. Then, by Lemma 3.4 and $(V .2)$, we have $\lambda+g \sup _{Q_{j \eta}}|V|-(1+2 \varepsilon) \Lambda_{l}^{ \pm} \geqslant g\left(\sup _{Q_{j \eta}}|V|\right.$ $-(1-\varepsilon) V(\bar{z})) \geqslant g\left(\varepsilon-C g^{-(\alpha-2 \sigma)}\right) V(\bar{z})>0$ for large $g>0$. This shows the claim. So, for large $g>0$, the rhs of (3.14) is bounded from below by

$$
\begin{aligned}
& (1-\varepsilon)^{2} \frac{B_{ \pm}}{2 \pi} \sum_{l \in \mathbf{N}} \sum_{j \in J_{ \pm}^{\prime}} \operatorname{Vol}\left(Q_{j}\right) \\
& \geqslant(1-\varepsilon)^{2} \frac{B_{ \pm}}{2 \pi} \sum_{l \in N} \operatorname{Vol}\left(\left(\Omega_{ \pm} \cap \Omega_{ \pm}^{(-)}(\varepsilon, l)\right) \backslash\left\{z \in \Omega_{ \pm} \cap \Omega_{ \pm}^{(-)}(\varepsilon, l) \mid \operatorname{dist}\left(z, \partial \Omega_{ \pm}\right) \leqslant g^{\sigma}\right\}\right) \\
& \geqslant(1-\varepsilon)^{2} \frac{B_{ \pm}}{2 \pi} \sum_{l \in \mathbf{N}} \operatorname{Vol}\left(\Omega_{ \pm} \cap \Omega_{ \pm}^{(-)}(\varepsilon, l)\right)-(1-\varepsilon)^{2} \frac{B_{ \pm}}{2 \pi} \sum_{l=0}^{C_{g^{1-m \alpha}}} \operatorname{Vol}\left\{z \in \Omega_{ \pm} \cap \Omega_{ \pm}^{(-)}(\varepsilon, l) \mid \operatorname{dist}\left(z, \partial \Omega_{ \pm}\right)\right. \\
& \left.\quad \leqslant g^{\sigma}\right\},
\end{aligned}
$$

where we used the fact that $\left(\Omega_{ \pm} \cap \Omega_{ \pm}^{(-)}(\varepsilon, l)\right) \backslash\left\{z \in \Omega_{ \pm} \cap \Omega_{ \pm}^{(-)}(\varepsilon, l) \mid \operatorname{dist}\left(z, \partial \Omega_{ \pm}\right) \leqslant g^{\sigma}\right\} \subset \cup_{j \in J_{ \pm}^{\prime}} Q_{j}$, where the rhs is a disjoint union, in the first inequality and used, in the last inequality, the fact that the set $\Omega_{ \pm} \cap \Omega_{ \pm}^{(-)}(\varepsilon, l)=\left\{z \in \Omega_{ \pm} \mid(1+2 \varepsilon) \Lambda_{l}^{ \pm}<\lambda+(1-\varepsilon) g V(z)\right\}$ is empty for all $l$ satisfying (1 $+2 \varepsilon) \Lambda_{l}^{ \pm} \geqslant \bar{\lambda}+C g^{1-m \alpha}$ since $\sup _{\Omega_{ \pm}}|V| \leqslant C\left\langle g^{\alpha}-g^{\sigma}\right\rangle^{-m} \leqslant C g^{-m \alpha}$ holds for large $g$. 
From geometry, we have $\operatorname{Vol}\left(\Omega_{0} \cap \Omega_{ \pm}^{(-)}(\varepsilon, l)\right) \leqslant \operatorname{Vol} \Omega_{0} \leqslant C g^{\alpha+\beta}=o\left(g^{2 / m}\right)$ and $\operatorname{Vol}\left(\Omega_{\mp}\right.$ $\left.\cap \Omega_{ \pm}^{(-)}(\varepsilon, l)\right)=\operatorname{Vol}\left(\Omega_{1} \cap \Omega_{ \pm}^{(-)}(\varepsilon, l)\right)=0$ as $g \rightarrow \infty$, where we used the fact that $\Omega_{1} \subset\left\{z \in \mathbf{R}^{2}|| z \mid\right.$ $\left.\geqslant g^{\not{B}}\right\} \subset\left\{z \in \mathbf{R}^{2}|| z \mid \geqslant 2 C g^{1 / m}\right\}$ and $\Omega_{ \pm}^{(-)} \subset\left\{z \in \mathbf{R}^{2} \mid B_{-}<\lambda+C g\langle z\rangle^{-m}\right\} \subset\left\{z \in \mathbf{R}^{2}|| z \mid \leqslant C g^{1 / m}\right\}$ for some $C>0$. Hence, we have

$$
\operatorname{Vol}\left(\Omega_{ \pm} \cap \Omega_{ \pm}^{(-)}(\varepsilon, l)\right)=\operatorname{Vol}\left(\Omega_{ \pm}^{(-)}(\varepsilon, l)\right)+o\left(g^{2 / m}\right)
$$

as $g \rightarrow \infty$, and we observe that

$$
\sum_{l=0}^{C g^{1-m \alpha}} \operatorname{Vol}\left\{z \in \Omega_{ \pm} \cap \Omega_{ \pm}^{(-)}(\varepsilon, l) \mid \operatorname{dist}\left(z, \partial \Omega_{ \pm}\right) \leqslant g^{\sigma}\right\} \leqslant C g^{1-m \alpha} g^{\beta+\sigma}=o\left(g^{2 / m}\right)
$$

as $g \rightarrow \infty$, since $\Omega_{ \pm} \cap \Omega_{ \pm}^{(-)}(\varepsilon, l) \subset \Omega_{ \pm}$and $1-m \alpha+\beta+\sigma=2 / m-(1 / m-2(m+1) \sigma)<2 / m$. Then the lemma follows from (3.14)-(3.17).

Lemma 3.8: Let $\lambda<B_{-}$. Under the same assumption on $B$ and $V$ as in Theorem 1.1, we have $\lim _{g \rightarrow \infty} g^{-2 / m} N_{g}^{+}(\lambda)=\Sigma_{l \in \mathbf{N}} \nu_{l}(\lambda)$.

Proof: We can deduce from (3.11) and (3.12) and Lemma 3.7 that $\lim _{\inf } g_{g \rightarrow \infty} g^{-2 / m} N_{g}^{+}(\lambda)$ $\geqslant \Sigma_{l \in \mathrm{N}} \nu_{l}(\lambda)$ in the same way as in the proof of Lemma 3.6. Then, combining this and Lemma 3.6, we have the result.

Remark 3.9: Our proof shows that we can replace the assumptions on B and $V$ in Lemma 3.8 by the following weaker assumptions.

$(B)^{\prime}$ In addition to (B.1), there exist $M>0$ and $C>0$ such that $\left|B(x)-B_{ \pm}\right| \leqslant C\langle x\rangle^{-M}$ hold as $x \rightarrow \pm \infty$, respectively.

$(V)^{\prime}$ The non-negative scalar potential $V$ belongs to $C^{1}(\mathbf{R})$, and there exist $m>0$ and $C>0$ such that $0<m<2$ and $\left|\partial^{\alpha} V(z)\right| \leqslant C\langle z\rangle^{-m-|\alpha|}$ holds for all $z \in \mathbf{R}^{2}$ and for any multi-index $\alpha$ satisfying $|\alpha| \leqslant 1$. Moreover, the conditions (V.2) and (V.3) hold.

\section{SPECTRAL PROPERTIES OF THE IWATSUKA MODEL}

\section{A. Direct decomposition}

In this section we recall from Iwatsuka (1985) the spectral representation of the Iwatsuka $H_{0}(b)$. We introduce the partial Fourier transform $(\mathcal{F} u)(x, \eta)=(2 \pi)^{-1 / 2} \int_{\mathbf{R}} e^{-\sqrt{-1} y} u(x, y) \mathrm{d} y$, which defines a unitary operator on $L^{2}\left(\mathbf{R}^{2}\right)$. We write $L_{0}$ for $\mathcal{F} H_{0}(b) \mathcal{F}^{-1}$. Then $L_{0}$ has the direct integral decomposition $L_{0}=\int_{\mathbf{R}}^{\oplus} L(\eta) \mathrm{d} \eta$ acting on the Hilbert space $\int_{\mathbf{R}}^{\oplus} L^{2}(\mathbf{R}) \mathrm{d} \eta$. Here, for each $\eta \in \mathbf{R}$, the fiber $L(\eta)$ of $L_{0}$ is given by

$$
L(\eta)=-\frac{\mathrm{d}^{2}}{\mathrm{~d} x^{2}}+(\eta-b(x))^{2}
$$

acting on the fiber $L^{2}(\mathbf{R})$ of $\int_{\mathbf{R}}^{\oplus} L^{2}(\mathbf{R}) \mathrm{d} \eta$.

The spectral properties of $L(\eta)$ are summarized as follows.

Lemma 4.1: [Iwatsuka ${ }^{11}$, Lemmas 2.3 and 3.5)] Assume that (B.1) holds. For each $\eta \in \mathbf{R}$, the operator $L(\eta)$ is essentially self-adjoint on $C_{0}^{\infty}(\mathbf{R})$ and has a complete orthonormal system of eigenfunctions $\left\{\varphi_{n}(\cdot, \eta)\right\}_{n \in \mathbf{N}}$ and the corresponding eigenvalues $\left\{\lambda_{n}(\eta)\right\}_{n \in \mathbf{N}}$ so that $L(\eta) \varphi_{n}(\cdot, \eta)$ $=\lambda_{n}(\eta) \varphi_{n}(\cdot, \eta)$ and $0<\lambda_{0}(\eta)<\lambda_{1}(\eta)<\lambda_{2}(\eta)<\cdots \rightarrow \infty$ hold for each $n \in \mathbf{N}$.

Moreover, the following properties (1)-(5) hold for each $n \in \mathbf{N}$.

(1) $\lambda_{n}(\eta)$ is nondegenerate and depends analytically on $\eta$.

(2) $\lambda_{n}(\eta)$ is monotonically increasing in $\eta$, and $\lim _{\eta \rightarrow \pm \infty} \lambda_{n}(\eta)=\Lambda_{n}^{ \pm}$hold, respectively.

(3) $\varphi_{n}(\cdot, \eta)$ belongs to $D(L(0))$ and depends analytically on $\eta$ with respect to the graph norm $\left(\|u\|^{2}+\|L(0) u\|^{2}\right)^{1 / 2}$.

(4) $\varphi_{n}(x, \eta)$ is a real-valued continuous function of $x$ and $\eta$, and moreover, $\varphi_{n}(x, \eta)$ is infinitely differentiable in $x$ for each $\eta$ and is analytic in $\eta$ for each $x$.

(5) The estimate $\left|\varphi_{n}(x, \eta)\right| \leqslant \Phi_{n}\left(x-b^{-1}(\eta)\right)$ holds for a function $\Phi_{n}$ satisfying $0<\Phi_{0}(x)<\cdots$ 


$$
\begin{aligned}
& <\Phi_{n}(x)<\cdots \text { and } \\
& \qquad \Phi_{n}(x) \leqslant \begin{cases}\sqrt{2}\left(\Lambda_{n}^{+}\right)^{1 / 4} & \text { if }|x| \leqslant \sqrt{\Lambda_{n}^{+}} / B_{-}, \\
\sqrt{2}\left(\Lambda_{n}^{+}\right)^{1 / 4} \exp \left\{-B_{-}\left(|x|-L_{n}\right)^{2} / 2\right\} & \text { if }|x| \geqslant \sqrt{\Lambda_{n}^{+}} / B_{-} .\end{cases}
\end{aligned}
$$

The next result follows easily from $(B .1)$ and the definition of $b$.

Lemma 4.2: Under the assumption (B.1), the function $b$ has the inverse $b^{-1}$ and moreover, for any $x, y, \eta$, we have $B_{-}\left|x-b^{-1}(\eta)\right| \leqslant|b(x)-\eta| \leqslant B_{+}\left|x-b^{-1}(\eta)\right|$ and $B_{-}|x-y| \leqslant|b(x)-b(y)| \leqslant B_{+} \mid x$ $-y \mid$.

For any $k \in \mathbf{N}$, we introduce the Banach space $B^{k}=\left\{u \in \mathcal{S}^{\prime}(\mathbf{R}) \mid x^{\alpha} \partial_{x}^{\beta} u \in L^{2}(\mathbf{R})\right.$ if $\left.\alpha+\beta \leqslant k\right\}$ with norm $\|u\|_{B^{k}}=\left(\Sigma_{\alpha+\beta \leqslant k}\left\|x^{\alpha} \partial_{x}^{\beta} u\right\|^{2}\right)^{1 / 2}$.

Lemma 4.3: Let $D_{0}$ denote either $C_{0}^{\infty}(\mathbf{R})$ or $\mathcal{S}(\mathbf{R})$. We have the following assertions.

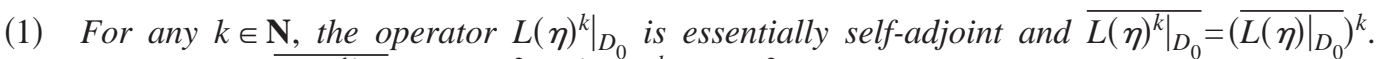
Moreover, $D\left(\overline{\left.L(\eta)^{k}\right|_{D_{0}}}\right)=\left\{u \in L^{2}(\mathbf{R}) \mid L(\eta)_{\text {dist }}^{k} u \in L^{2}(\mathbf{R})\right\}$. Here, $L_{\text {dist }}$ stands for the differential operator $L$ with domain $\mathcal{S}^{\prime}(\mathbf{R})$.

(2) The Banach space $D\left(\overline{\left.L(\eta)^{k}\right|_{D_{0}}}\right)$ equipped with the graph norm $\overline{\left.L(\eta)^{k}\right|_{D_{0}}}$ coincides with the Banach space $D\left(\overline{\left.L(0)^{k}\right|_{D_{0}}}\right)$ equipped with (equivalent) norm by $\overline{\left.L(0)^{k}\right|_{D_{0}}}$.

(3) If we denote by $\widetilde{B}^{k}$ the space as in the assertion (2) above, then $\widetilde{B}^{k}$ coincides with $B^{k}$ as a Banach space. In particular, the space $\cap_{k \in \mathbf{N}} \widetilde{B}^{k}$ coincides with $\mathcal{S}(\mathbf{R})$ as a Fréchet space.

Proof: We note that $L(\eta) \in O p S^{2}$, so $L(\eta)^{k} \in O p S^{2 k}$ for any $\eta$. It is easy to see that the symbol of $L(\eta)^{k}$ satisfies the ellipticity condition as in Lemma 2.7 and $L(\eta)$ is symmetric on $D_{0}$. Then, by Lemma 2.7, the operator $\left.L(\eta)^{k}\right|_{D_{0}}$ is essentially self-adjoint and $D\left(\overline{\left.L(\eta)^{k}\right|_{D_{0}}}\right)=\{u$ $\left.\in L^{2}(\mathbf{R}) \mid L(\eta)_{\text {dist }}^{k} u \in L^{2}(\underline{\mathbf{R}})\right\}$. Also, we find that $\left.L(\eta)^{k}\right|_{D_{0}}=\left(\left.\left.\overline{L(\eta)}\right|_{D_{0}}{ }^{k}\right|_{D_{0}}\right.$ since $L(\eta)$ leaves $D_{0}$ invariant. This implies that $\overline{\left.L(\eta)^{k}\right|_{D_{0}}} \subset\left(\overline{\left.L(\eta)\right|_{D_{0}}}\right)^{k}$, which are both self-adjoint, so coincide. This shows the assertion (1).

The assertion (2) follows from Lemma 2.7 with $O p(a)=L(\eta)^{k}$ and with $O p(a)=L(0)^{k}$ since the topology of the weighted Sobolev spaces $H^{m}$ is independent of specific $O p(a)$ by the (original) definition. As a byproduct, we find that $D\left(\overline{\left.L(\eta)^{k}\right|_{D_{0}}}\right)$ coincides with $H^{2 k}$.

Finally we show the assertion (3). It is enough to show in the case of $\eta=0$. Note that $B_{-}|x|$ $\leqslant|b(x)| \leqslant B_{+}|x|$ holds for all $x \in \mathbf{R}$ by Lemma 4.2 , and each derivative of $b$ is bounded. Then there exists $C_{k}>0$ such that

$$
\left\|L(0)^{k} u\right\|^{2}+\|u\|^{2} \leqslant C_{k}\|u\|_{B^{2 k}}^{2}
$$

holds for any $u \in D_{0}$. Conversely, by the assertion (4) in Lemma 2.6, the operator $x^{\beta} \partial_{x}^{\alpha}$ maps $H^{2 k}$, which coincides with $D\left(\overline{\left.L(\eta)^{k}\right|_{D_{0}}}\right)$ as stated above, to $L^{2}(\mathbf{R})$ continuously provided $\alpha+\beta \leqslant 2 k$. This means the inequality $\left\|x^{\beta} \partial_{x}^{\alpha} u\right\| \leqslant C\left(\left\|L(0)^{k} u\right\|+\|u\|\right)$ for any $u \in D_{0}$. The density of $D_{0}$ completes the proof.

\section{B. Exponential decay of $\varphi_{n}$}

In this section, using the so-called Agmon estimate, we derive an exponential decay estimates of the eigenfunction $\varphi_{n}$ and obtain the estimates for the band function $\lambda_{n}$. To the end of this section, we set $g(x, \eta)=\left(x-b^{-1}(\eta)\right)^{2}$ for any $x, \eta \in \mathbf{R}$.

Lemma 4.4: Let $\eta \in \mathbf{R}$ and let $L(\eta)$ be as in (4.1). Assume that $f \in L^{2}(\mathbf{R})$ and there exists $\kappa>0$ such that $\left\|e^{\kappa g(\cdot, \eta)} f\right\|$ is finite. Assume that $\psi$ in $D(L(\eta))$ satisfies the equation $(L(\eta)$ $\left.-\lambda_{n}(\eta)\right) \psi=f$. Then there exists $C_{n}=C_{n}\left(\kappa, B_{-}\right)>0$, independent of $\eta$, such that

$$
\left\|e^{\alpha g(\cdot, \eta)} \psi\right\| \leqslant C_{n}\left(\left\|e^{\kappa g(\cdot, \eta)} f\right\|+\|\psi\|\right)
$$

holds if $0<\alpha<\min \left\{\kappa, B_{-}^{2} / \sqrt{8}\right\}$.

Proof: This is an easy consequence of the method of Agmon estimates. However, we give a 
proof for the sake of completeness. Let $\chi \in C^{\infty}([0, \infty))$ such that $\chi(t)=t$ if $0 \leqslant t \leqslant 1 / 2, \chi(t)=1$ if $t \geqslant 2, \sup _{t \geqslant 0}\left|\chi^{\prime}(t)\right| \leqslant 1$ and $\chi$ is monotonically increasing. For any large $R>0$, we set $g_{R}(x, \eta)$ $=R^{2} \chi\left(g(x, \eta) / R^{2}\right)$. We can find that

$$
\left|g_{R}(x, \eta)\right| \leqslant \min \left\{R^{2}, g(x, \eta)\right\}, \quad\left|\partial_{x} g_{R}(x, \eta)\right| \leqslant 2\left|x-b^{-1}(\eta)\right|
$$

and $\lim _{R \rightarrow \infty} g_{R}(x, \eta)=g(x, \eta)$ for any $(x, \eta)$.

We may assume that $\psi$ is not identically zero. The standard Agmon-type argument shows that

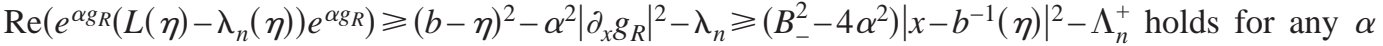
$\in \mathbf{R}$, where we used Lemma 4.2 in the first inequality and "Re" stands for the real part. Then it follows that

$$
\left\|e^{\alpha g_{R}} \psi\right\|\left\|e^{\alpha g_{R}} f\right\| \geqslant\left|\operatorname{Re}\left(e^{2 \alpha g_{R}} \psi, f\right)\right| \geqslant\left(e^{\alpha g_{R}} \psi,\left(\left(B_{-}^{2}-4 \alpha^{2}\right)\left|x-b^{-1}\right|^{2}-\Lambda_{n}^{+}\right) e^{\alpha g_{R}} f\right) .
$$

Take $\phi \in C_{0}^{\infty}([0, \infty))$ so that $\phi(t)=1$ if $0 \leqslant t \leqslant 1, \phi(t)=0$ if $t \geqslant 2$ and $\phi$ is monotonically decreasing. Set $\phi_{\eta}(x)=\phi\left(B_{-}^{2} g(x, \eta) /\left(2 \Lambda_{n}^{+}\right)\right)$. Then we find that

$$
\left(B_{-}^{2}-4 \alpha^{2}\right)\left|x-b^{-1}\right|^{2}-\Lambda_{n}^{+} \geqslant\left(1-8 \alpha^{2} / B_{-}^{2}\right) \Lambda_{n}^{+}-6 \Lambda_{n}^{+} \phi_{\eta}
$$

using the fact that $g(x, \eta) \leqslant 4 \Lambda_{n}^{+} / B_{-}^{2}$ holds on supp $\phi_{\eta}$ and $g(x, \eta) \geqslant 2 \Lambda_{n}^{+} / B_{-}^{2}$ holds on supp (1 $\left.-\phi_{\eta}\right)$, and we find from (4.4) and (4.5) that $\left(1-8 \alpha^{2} / B_{-}^{2}\right) \Lambda_{n}^{+}\left\|e^{\alpha g_{R}} \psi\right\| \leqslant\left\|e^{\alpha g_{R}} f\right\|+6 \Lambda_{n}^{+} e^{4 \alpha \Lambda_{n}^{+} / B_{-}^{2}}\|\psi\|$, since $g_{R}(x, \eta) \leqslant g(x, \eta) \leqslant 4 \Lambda_{n}^{+} / B_{-}^{2}$ holds on supp $\phi_{\eta}$. Taking a limit $R \rightarrow \infty$, we have the conclusion.

Lemma 4.5: Let $\alpha \in \mathbf{N}$. There exists $\kappa=\kappa_{\alpha, n}>0$ such that

$$
\sup _{\eta \in \mathbf{R}}\left\|e^{\kappa g} \partial_{\eta}^{\alpha^{\prime}} \varphi_{n}(\eta)\right\|<\infty \quad \text { and } \sup _{\eta \in \mathbf{R}}\left|\partial_{\eta}^{\alpha^{\prime}+1} \lambda_{n}(\eta)\right|<\infty
$$

hold if $0 \leqslant \alpha^{\prime} \leqslant \alpha$.

Proof: We show this by induction on $\alpha$. We abbreviate the assertion to $P(\alpha)$. When $\alpha=0$, the first estimate in (4.6) follows from Lemma 4.1 (v). The Feynman-Hellman formula yields that

$$
\partial_{\eta} \lambda_{n}(\eta)=\left(\varphi_{n},\left(\partial_{\eta} L(\eta)\right) \varphi_{n}\right)=2\left(\varphi_{n},(\eta-b) \varphi_{n}\right)
$$

Then the second estimate in (4.6) follows from the exponential decay of $\varphi_{n}$ with respect to $\eta$ $-b$. This shows that $P(0)$ is true.

We assume that $P(\alpha)$ is true. First, under this assumption, we show the estimate

$$
\sup _{\eta \in \mathbf{R}}\left\|\partial_{\eta}^{\alpha+1} \varphi_{n}(\eta)\right\|<\infty
$$

Differentiating the equation $\left(L(\eta)-\lambda_{n}(\eta)\right) \varphi_{n}(\eta)=0$ with respect to $\eta$ in $(\alpha+1)$ times, we obtain

$$
\left(L(\eta)-\lambda_{n}(\eta)\right) \partial_{\eta}^{\alpha+1} \varphi_{n}(\eta)=-\sum_{0 \leqslant \alpha^{\prime} \leqslant \alpha} C_{\alpha+1, \alpha^{\prime}} \partial_{\eta}^{\alpha+1-\alpha^{\prime}}\left((b-\eta)^{2}-\lambda_{n}(\eta)\right) \partial_{\eta}^{\alpha^{\prime}} \varphi_{n}(\eta)
$$

In the case of $j \neq n$, taking an inner product with $\varphi_{j}$, we obtain

$$
\left(\lambda_{j}(\eta)-\lambda_{n}(\eta)\right)\left(\partial_{\eta}^{\alpha+1} \varphi_{n}, \varphi_{j}\right)=-\sum_{0 \leqslant \alpha^{\prime} \leqslant \alpha} C_{\alpha+1, \alpha^{\prime}}\left(\partial_{\eta}^{\alpha+1-\alpha^{\prime}}\left((b-\eta)^{2}-\lambda_{n}(\eta)\right) \partial_{\eta}^{\alpha^{\prime}} \varphi_{n}, \varphi_{j}\right)
$$

Then the Schwarz inequality yields that 


$$
\begin{aligned}
\left|\left(\partial_{\eta}^{\alpha+1} \varphi_{n}, \varphi_{j}\right)\right|^{2} & \leqslant\left(C_{\alpha} \delta_{n}^{-1} \sum_{0 \leqslant \alpha^{\prime} \leqslant \alpha}\left|\left(\partial_{\eta}^{\alpha+1-\alpha^{\prime}}\left((b-\eta)^{2}-\lambda_{n}(\eta)\right) \partial_{\eta}^{\alpha^{\prime}} \varphi_{n}, \varphi_{j}\right)\right|\right)^{2} \\
& \leqslant C_{\alpha}^{\prime} \delta_{n}^{-2} \sum_{0 \leqslant \alpha^{\prime} \leqslant \alpha}\left|\left(\partial_{\eta}^{\alpha+1-\alpha^{\prime}}\left((b-\eta)^{2}-\lambda_{n}(\eta)\right) \partial_{\eta}^{\alpha^{\prime}} \varphi_{n}, \varphi_{j}\right)\right|^{2}
\end{aligned}
$$

for some $C_{\alpha}, C_{\alpha}^{\prime}>0$ independent of $\eta$. Here, we introduced a positive number $\delta_{n}$ as

$$
\delta_{n}=\left\{\begin{array}{c}
\min \left\{\inf _{\eta \in \mathbf{R}}\left(\lambda_{n+1}(\eta)-\lambda_{n}(\eta)\right), \inf _{\eta \in \mathbf{R}}\left(\lambda_{n}(\eta)-\lambda_{n-1}(\eta)\right)\right\} \quad \text { if } n \geqslant 2, \\
\inf _{\eta \in \mathbf{R}}\left(\lambda_{2}(\eta)-\lambda_{1}(\eta)\right) \text { if } n=1 .
\end{array}\right.
$$

In the case of $j=n$, differentiating the relation $\left(\varphi_{n}, \varphi_{n}\right)=1$ with respect to $\eta$ in $(\alpha+1)$ times, we find that $2\left(\partial_{\eta}^{\alpha+1} \varphi_{n}, \varphi_{n}\right)=-\Sigma_{1 \leqslant \alpha^{\prime} \leqslant \alpha} C_{\alpha+1, \alpha^{\prime}}\left(\partial_{\eta}^{\alpha+1-\alpha^{\prime}} \varphi_{n}, \partial_{\eta}^{\alpha^{\prime}} \varphi_{n}\right)$, where we used the reality of $\varphi_{n}$. Then we have

$$
\left|\left(\partial_{\eta}^{\alpha+1} \varphi_{n}, \varphi_{n}\right)\right| \leqslant C_{\alpha} \sum_{1 \leqslant \alpha^{\prime} \leqslant \alpha}\left\|\partial_{\eta}^{\alpha+1-\alpha^{\prime}} \varphi_{n}\right\|\left\|\partial_{\eta}^{\alpha^{\prime}} \varphi_{n}\right\|
$$

for some $C_{\alpha}>0$ independent of $\eta$. Hence it follows from (4.10) and (4.11) that

$$
\begin{aligned}
\left\|\partial_{\eta}^{\alpha+1} \varphi_{n}\right\|^{2}= & \sum_{j \in N}\left|\left(\partial_{\eta}^{\alpha+1} \varphi_{n}, \varphi_{j}\right)\right|^{2} \leqslant C_{\alpha}^{\prime} \delta_{n}^{-2} \sum_{0 \leqslant \alpha^{\prime} \leqslant \alpha}\left\|\left(\partial_{\eta}^{\alpha+1-\alpha^{\prime}}\left((b-\eta)^{2}-\lambda_{n}(\eta)\right)\right) \partial_{\eta}^{\alpha^{\prime}} \varphi_{n}\right\|^{2} \\
& +\left(C_{\alpha} \sum_{1 \leqslant \alpha^{\prime} \leqslant \alpha}\left\|\partial_{\eta}^{\alpha+1-\alpha^{\prime}} \varphi_{n}\right\|\left\|\partial_{\eta}^{\alpha^{\prime}} \varphi_{n}\right\|\right)^{2},
\end{aligned}
$$

where we used the fact that $\left\{\varphi_{j}(\cdot, \eta)\right\}_{j \in \mathbf{N}}$ is an ONB in $L^{2}(\mathbf{R})$. By the assumption $P(\alpha)$, all terms on the rhs of (4.12) are bounded uniformly in $\eta$. Thus we have proved (4.8) under $P(\alpha)$.

Next, we apply Lemma 4.4 to (4.9) with $\psi=\partial_{\eta}^{\alpha+1} \varphi_{n}, f=f_{\alpha}=$ the rhs of (4.9). We may assume that the constant $\alpha$ in the exponent in Lemma 4.4 is equal to $\kappa$ since we can take $\kappa$ small enough. Then it follows that there exists $C_{n}>0$ such that the estimate $\left\|e^{\kappa g} \partial_{\eta}^{\alpha+1} \varphi_{n}\right\| \leqslant C_{n}\left(\left\|e^{\kappa g} f_{\alpha}\right\|\right.$ $\left.+\left\|\partial_{\eta}^{\alpha+1} \varphi_{n}\right\|\right)$. The first term on the rhs is bounded uniformly in $\eta$ under $P(\alpha)$, since the maximal order of derivatives of $\varphi_{n}$ is less than or equal to $\alpha$ and the maximal order of derivatives of $\lambda_{n}$ is less than or equal to $\alpha+1$ in the expression of $f_{\alpha}$. The second term on the rhs is also uniformly bounded because of (4.8) we have already proved. Hence, we have proved the first estimate in (4.6) for $P(\alpha+1)$, assuming $P(\alpha)$.

Finally, we show the second estimate in (4.6) for $P(\alpha+1)$, assuming $P(\alpha)$. Differentiating (4.7) with respect to $\eta$ in $(\alpha+1)$ times, we obtain

$$
\partial_{\eta}^{\alpha+2} \lambda_{n}(\eta)=2 \partial_{\eta}^{\alpha+1}\left(\varphi_{n},(\eta-b) \varphi_{n}\right)=2 \sum_{0 \leqslant \alpha^{\prime} \leqslant \alpha+1} C_{\alpha+1, \alpha^{\prime}}\left(\partial_{\eta}^{\alpha+1-\alpha^{\prime}} \varphi_{n}, \partial_{\eta}^{\alpha^{\prime}}\left((\eta-b) \varphi_{n}\right)\right) .
$$

Since the maximal order of derivatives of $\varphi_{n}$ on the rhs of (4.13) is less than or equal to $\alpha+1$, the uniform boundedness of the rhs of (4.13) with respect to $\eta$ follows from the first estimate in (4.6) for $P(\alpha)$ and the exponential decay property of $\partial_{\eta}^{\alpha+1} \varphi_{n}$ we have proved above. Thus we have proved all the assertion of $P(\alpha+1)$, assuming $P(\alpha)$. This completes the induction.

Lemma 4.6: Let $\beta \in \mathbf{N}$. For any $\alpha \in \mathbf{N}$, there exists $\kappa=\kappa(\alpha, \beta, n)>0$ such that $\sup _{\eta \in \mathbf{R}}\left\|e^{\kappa g} \partial_{x}^{\beta} \partial_{\eta}^{\alpha} \varphi_{n}(\eta)\right\|<\infty$.

Proof: We show this by induction on $\beta$. We abbreviate the assertion to $P(\beta)$. The assertion $P(0)$ is true by Lemma 4.5 .

We assume that $P(\beta)$ is true. Then, for any $\alpha$, we have

$$
\begin{aligned}
\left\|e^{\kappa g} \partial_{x}^{\beta+1} \partial_{\eta}^{\alpha} \varphi_{n}(\eta)\right\|^{2}= & -\left(e^{2 \kappa g} \partial_{x}^{\beta} \partial_{\eta}^{\alpha} \partial_{x}^{2} \varphi_{n}, \partial_{x}^{\beta} \partial_{\eta}^{\alpha} \varphi_{n}\right)-4 \kappa\left(\left(x-b^{-1}(\eta)\right) e^{2 \kappa g} \partial_{x}^{\beta+1} \partial_{\eta}^{\alpha} \varphi_{n}, \partial_{x}^{\beta} \partial_{\eta}^{\alpha} \varphi_{n}\right) \\
= & \left(e^{2 \kappa g} \partial_{x}^{\beta} \partial_{\eta}^{\alpha}\left(\lambda_{n}(\eta)-(b-\eta)^{2}\right) \varphi_{n}, \partial_{x}^{\beta} \partial_{\eta}^{\alpha} \varphi_{n}\right) \\
& -4 \kappa\left(\partial_{x}^{\beta+1} \partial_{\eta}^{\alpha} \varphi_{n},\left(x-b^{-1}(\eta)\right) e^{2 \kappa g} \partial_{x}^{\beta} \partial_{\eta}^{\alpha} \varphi_{n}\right),
\end{aligned}
$$


where we used the equation $\left(L-\lambda_{n}\right) \varphi_{n}=0$ in the third equality. The first term on the rhs of (4.14) is bounded uniformly in $\eta$ by the assumption $P(\beta)$ and the second estimate in (4.6) if we choose $\kappa>0$ sufficiently small (the choice is independent of $\eta$ ). The second term on the rhs of (4.14) is bounded from above by $4 \kappa\left\|e^{\kappa g} \partial_{x}^{\beta+1} \partial_{\eta}^{\alpha} \varphi_{n}\right\|\left\|\left(x-b^{-1}(\eta)\right) e^{\kappa g} \partial_{x}^{\beta} \partial_{\eta}^{\alpha} \varphi_{n}\right\| \leqslant 2 \kappa\left\|e^{\kappa g} \partial_{x}^{\beta+1} \partial_{\eta}^{\alpha} \varphi_{n}\right\|^{2}+2 \kappa \|(x$ $\left.-b^{-1}\right) e^{\kappa g} \partial_{\beta} \partial_{\eta}^{\alpha} \varphi_{n} \|^{2}$, which is uniformly bounded since $P(\beta)$ is assumed to be true.

Lemma 4.7: For any $n, \alpha, \beta \in \mathbf{N}$, there exists a positive constant $\kappa=\kappa(\alpha, \beta, n)$ such that $\sup _{(x, \eta) \in \mathbf{R}^{2}}\left|e^{\kappa g} \partial_{x}^{\beta} \partial_{\eta}^{\alpha} \varphi_{n}(x, \eta)\right|<\infty$ holds.

Proof: For any $\gamma \in \mathbf{N}$, we have $\partial_{x}^{\gamma}\left(e^{\kappa g} \partial_{x}^{\beta} \partial_{\eta}^{\alpha} \varphi_{n}\right)=\Sigma_{0 \leqslant \gamma^{\prime} \leqslant \gamma} C_{\gamma, \gamma^{\prime}}\left(\partial_{x}^{\gamma-\gamma^{\prime}} e^{\kappa g}\right)\left(\partial_{x}^{\beta+\gamma^{\prime}} \partial_{\eta}^{\alpha} \varphi_{n}\right)$. Since each of the functions $\partial_{x}^{\gamma-\gamma^{\prime}} e^{\kappa g}$ is of the form "a polynomial of $\left(x-b^{-1}(\eta)\right)$ times $e^{\kappa g}$," we obtain the estimate

$$
\left\|\partial_{x}^{\gamma}\left(e^{\kappa g} \partial_{x}^{\beta} \partial_{\eta}^{\alpha} \varphi_{n}\right)\right\| \leqslant \sum_{0 \leqslant \gamma^{\prime} \leqslant \gamma} C_{\gamma, \gamma^{\prime}}\left\|e^{\kappa^{\prime} g} \partial_{x}^{\beta+\gamma^{\prime}} \partial_{\eta}^{\alpha} \varphi_{n}\right\|
$$

for some $\kappa^{\prime}>0$ satisfying $0<\kappa^{\prime}<\kappa$ uniformly in $\eta$. The rhs of (4.15) is bounded uniformly in $\eta$ by Lemma 4.6 for an appropriate choice of $\kappa, \kappa^{\prime}$. This means that the $\gamma$ th Sobolev norm of $e^{\kappa g} \partial_{x}^{\beta} \partial_{\eta}^{\alpha} \varphi_{n}$ is bounded uniformly in $\eta$ for each $\gamma$. Hence, Sobolev's embedding theorem yields that

$$
\sup _{x \in \mathbf{R}}\left|e^{\kappa g} \partial_{x}^{\beta} \partial_{\eta}^{\alpha} \varphi_{n}\right| \leqslant C\left(\left\|e^{\kappa g} \partial_{x}^{\beta} \partial_{\eta}^{\alpha} \varphi_{n}\right\|+\left\|\partial_{x}\left(e^{\kappa g} \partial_{x}^{\beta} \partial_{\eta}^{\alpha} \varphi_{n}\right)\right\|\right)
$$

for some $C>0$. The above observation shows that the rhs of (4.16) is uniformly bounded in $\eta$ if we choose $\kappa$ small enough. This shows the lemma.

\section{Decay estimate of $\lambda_{n}$}

In this section, we derive the following decay estimate of the band function $\lambda_{n}$ at infinity.

Lemma 4.8: Assume that (B.2) $)_{+}$holds. Then, for any $n \in \mathbf{N}$, there exists $C_{n}>0$ such that $\left|\Lambda_{n}^{+}-\lambda_{n}(b(x))\right| \leqslant C_{n}\langle x\rangle^{-M}$ holds if $x \geqslant 0$. Similarly, if we assume (B.2)_then the same estimate replaced $\Lambda_{n}^{+}$by $\Lambda_{n}^{-}$holds if $x \leqslant 0$.

Proof: We mimic the proof of Lemma 4.1 in Iwatsuka (1985). We first consider the case of $x \geqslant 0$. Let $n$ be fixed and $j, k \leqslant n$. Set $L_{c}(s)=-\partial_{x}^{2}+B_{+}^{2}(x-s)^{2}$ in $L^{2}(\mathbf{R})$ and $\alpha_{j k}(s)$ $=\left(L_{c}(s) \varphi_{j}(\cdot, b(s)), \varphi_{k}(\cdot, b(s))\right)$. Then, using the equation $\left(L(b(s)) \varphi_{j}(\cdot, b(s)), \varphi_{k}(\cdot, b(s))\right)$ $=\lambda_{j}(b(s)) \delta_{j k}$, we find that

$$
\begin{aligned}
\left|\alpha_{j k}(s)-\lambda_{j}(b(s)) \delta_{j k}\right| \leqslant & \int_{\mathbf{R}}\left|B_{+}^{2}(x-s)^{2}-(b(x)-b(s))^{2}\left\|\varphi_{j}(x, b(s))\right\| \varphi_{k}(x, b(s))\right| \mathrm{d} x \\
\leqslant & 2 B_{+} \int_{\mathbf{R}}\left\{|x-s|\left|\int_{s}^{x}\left(B_{+}-B(t)\right) \mathrm{d} t\right| \Phi_{n}(x-s)^{2}\right\} \mathrm{d} x \\
= & 2 B_{+} \int_{-\infty}^{-s / 2}\left\{|y|\left|\int_{s}^{y+s}\left(B_{+}-B(t)\right) \mathrm{d} t\right| \Phi_{n}(y)^{2}\right\} \mathrm{d} y \\
& +2 B_{+} \int_{-s / 2}^{\infty}\left\{|y|\left|\int_{s}^{y+s}\left(B_{+}-B(t)\right) \mathrm{d} t\right| \Phi_{n}(y)^{2}\right\} \mathrm{d} y,
\end{aligned}
$$

where we used the facts that $\left|B_{+}^{2}(x-s)^{2}-(b(x)-b(s))^{2}\right|=\left|\int_{s}^{x}\left(B_{+}+B(t)\right) \mathrm{d} t \| \int_{s}^{x}\left(B_{+}-B(t)\right) \mathrm{d} t\right| \leqslant 2 B_{+} \mid x$ $-s|| \int_{s}^{x}\left(B_{+}-B(t)\right) \mathrm{d} t \mid$ holds and that $\left|\varphi_{j}(x, b(s))\right| \leqslant \Phi_{n}(x-s)$ hold if $j \leqslant n$ by Lemma 4.1. For any large $N \in \mathbf{N}$ and any large $s>0$, the first term on the rhs of (4.17) is bounded from above by 


$$
\left(2 B_{+}\right)^{2} \int_{-\infty}^{-s / 2}|y|^{2} \Phi_{n}(y)^{2} \mathrm{~d} y \leqslant 4 B_{+}^{2} \int_{-\infty}^{-s / 2} C_{N, n}\langle y\rangle^{-2 N} \mathrm{~d} y \leqslant C_{N, n}^{\prime}\langle s\rangle^{N}
$$

for some constant $C_{N, n}^{\prime}$, where we used Lemma 4.1. Since $(B .2)_{+}$implies that there exists $s_{0}>0$ and $C_{n}>0$ such that $\sup _{t \geqslant s / 2}\left|B_{+}-B(t)\right| \leqslant C_{n}\langle s\rangle^{-N}$ holds if $s>s_{0}$, it follows that the second term on the rhs of (4.17) is bounded from above by

$$
2 B_{+} C_{n}\langle s\rangle^{-M} \int_{-s / 2}^{\infty}|y|^{2} \Phi_{n}(y)^{2} \mathrm{~d} y \leqslant C_{n}^{\prime} B_{+}\langle s\rangle^{-M}
$$

if $s>s_{0}$. Hence it follows from (4.17)-(4.19) that

$$
\sup _{s>s_{0}} s^{M}\left|\alpha_{j k}(s)-\lambda_{j}(b(s)) \delta_{j k}\right| \leqslant C_{n}
$$

holds for some $C_{n}>0$, if we choose $s_{0}$ sufficiently large.

If we denote by $V_{n}(s)$ the linear subspace of $L^{2}(\mathbf{R})$ spanned by $\left\{\varphi_{j}(\cdot, b(s))\right\}_{j \leqslant n}$, then $\left(\alpha_{j k}(s)\right)_{j, k \leqslant n}$ is the Hermitian symmetric matrix of $\left.L_{c}(s)\right|_{V_{n}(s)}$ with respect to the basis $\left\{\varphi_{j}(\cdot, b(s))\right\}_{j \leqslant n}$. Let $\mu_{0}(s) \leqslant \cdots \leqslant \mu_{n}(s)$ be the eigenvalues of $\left(\alpha_{j k}(s)\right)_{j, k \leqslant n}$. Let $\left(a_{i j}\right)$ and $\left(b_{i j}\right)$ be $n \times n$ Hermitian matrices and let $\alpha_{1} \leqslant \cdots \leqslant \alpha_{n}$ and $\beta_{1} \leqslant \cdots \leqslant \beta_{n}$ be their eigenvalues, respectively. Then it is easy to see that $\left|\alpha_{k}-\beta_{k}\right|^{2} \leqslant \Sigma_{i, j}\left|a_{i j}-b_{i j}\right|^{2}$ holds for any $k$. Hence it follows from (4.20) that

$$
\sup _{s>s_{0}} s^{M}\left|\mu_{j}(s)-\lambda_{j}(b(s))\right| \leqslant C_{n}^{\prime} .
$$

Then, by the variational principle [Reed and Simon (1978, Vol. IV, Theorem XIII.3)], we have $\Lambda_{j}^{+} \leqslant \mu_{j}(s)$ for any $j \leqslant n$. Since $\Lambda_{j}^{+}$is the $j$ th eigenvalue of $L_{c}(s)$ and $\lambda_{j}(b(s)) \leqslant \Lambda_{j}^{+}$, we can deduce from (4.21) that

$$
\sup _{s>s_{0}} s^{M}\left|\Lambda_{j}^{+}-\lambda_{j}(b(s))\right| \leqslant C_{n}^{\prime \prime}
$$

holds if $j \leqslant n$. This shows the first assertion of the lemma.

In the case of $s \rightarrow-\infty$, we have the conclusion in a similar way, replacing the objects $\alpha_{j k}, L_{c}(s), L(b(s))$ and $\mu_{j}(s)$ 's by $\left(L(b(s)) \psi_{j}(\cdot, s), \psi_{k}(\cdot, s)\right), L(b(s)),-\partial_{x}^{2}+B_{-}^{2}(x-s)^{2}$ and the eigenvalues of the matrix $\left(\left(L(b(s)) \psi_{j}(\cdot, s), \psi_{k}(\cdot, s)\right)\right)_{j, k \leqslant n}$, respectively. Here, $\psi_{k}(\cdot, s)$ is the eigenfunction of $-\partial_{x}^{2}+B_{-}^{2}(x-s)^{2}$ corresponding to the $k$ th eigenvalue $\Lambda_{k}^{-}$.

\section{PROOF OF THEOREMS}

\section{A. Operators on the direct sum}

To the end of this paper, we always assume (B.1), (B.2), (V.1)-(V.4).

Let $\left\{\varphi_{n}\right\}_{n \in \mathbf{N}}$ be the eigenfunctions given in Lemma 4.1. Because of Lemma 4.1, for any $l$ $\in \mathbf{N}$, we can define a partial isometry $T_{l}$ from $L^{2}(\mathbf{R})$ into $L^{2}\left(\mathbf{R}^{2}\right)$ by $\left(T_{l} f\right)(x, \eta)=\varphi_{l}(x, \eta) f(\eta)$ for any $f \in L^{2}(\mathbf{R})$. The adjoint operator $T_{l}^{*}$ from $L^{2}\left(\mathbf{R}^{2}\right)$ onto $L^{2}(\mathbf{R})$ is given by $\left(T_{l}^{*} F\right)(\eta)$ $=\int_{\mathbf{R}} \varphi_{l}(x, \eta) F(x, \eta) \mathrm{d} x$ for any $F \in L^{2}\left(\mathbf{R}^{2}\right)$. We now extend $T_{l} \mathrm{~s}$ to an operator on the direct sum of Hilbert spaces $\Sigma_{l \in \mathbf{N}} \oplus L^{2}(\mathbf{R})$, more precisely, we define a partial isometry $T$ from $\Sigma_{l \in \mathbf{N}} \oplus L^{2}(\mathbf{R})$ into $L^{2}\left(\mathbf{R}^{2}\right)$ by $T\left(\oplus_{l \in \mathbf{N}}\left(f_{l}\right)\right)=\Sigma_{l \in \mathbf{N}} T_{l} f_{l}$ for any $\oplus_{l \in \mathbf{N}}\left(f_{l}\right) \in \Sigma_{l \in \mathbf{N}} \oplus L^{2}(\mathbf{R})$. The unitarity of $T$ follows from the $L^{2}$-orthogonality of $\varphi_{l}$ 's with respect to the first variable. The adjoint operator $T^{*}$ from $L^{2}\left(\mathbf{R}^{2}\right)$ onto $\Sigma_{l \in \mathbf{N}} \oplus L^{2}(\mathbf{R})$ is given by $T^{*} F=\oplus_{l \in \mathbf{N}} T_{l}^{*} F$ for any $F \in L^{2}\left(\mathbf{R}^{2}\right)$. We set

$$
\mathcal{W}=T^{*} \mathcal{F} W \mathcal{F}^{*} T
$$

for any multiplication operator $W$ on $L^{2}\left(\mathbf{R}^{2}\right)$ and set $\mathcal{H}_{g}=T^{*} \mathcal{F} H_{g}(b) \mathcal{F}^{*} T\left(=\mathcal{H}_{0}-g \mathcal{V}\right)$, both acting on the Hilbert space $\Sigma_{l \in \mathbf{N}} \oplus L^{2}(\mathbf{R})$, where $\mathcal{F}$ is the partial Fourier transform as in the preceding section and $\mathcal{V}$ is the operator (5.1) with $W=V$. 
For an operator $A$ acting on the space $\Sigma_{l \in \mathbf{N}} \oplus L^{2}(\mathbf{R})$, we say $A$ belongs to $O p S^{m}$ if all the matrix elements $A_{j k}(j, k \in \mathbf{N})$ of $A$ (with respect to the direct sum decomposition) belong to the class $O p S^{m}$. When all the matrix-valued $\Psi$ DOs under consideration have finitely many nonzero components, the standard $\Psi$ DO calculus as in Sec. II B is applicable also for the matrix-valued

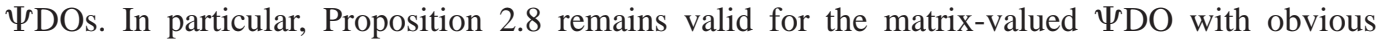
modifications (e.g., we regard the product of symbols as the usual matrix product, etc.).

In the rest of this section we are concerned with the matrix-valued $\Psi$ DO $\mathcal{V}=T^{*} \mathcal{F} V \mathcal{F}^{*} T$.

Lemma 5.1: Let $m>0$ be as in (V.1). We have the following assertions.

(1) The operator $T_{n}^{*} \mathcal{F} V \mathcal{F}^{*} T_{n}$ on $L^{2}(\mathbf{R})$ belongs to $O p S^{-m}$ for any $n \in \mathbf{N}$, and moreover, for any $\alpha, \beta \in \mathbf{N}$, there exists $C_{\alpha \beta}>0$ such that the Weyl symbol $p_{V}$ of $T_{n}^{*} \mathcal{F} V \mathcal{F}^{*} T_{n}$ on $L^{2}(\mathbf{R})$ satisfies the estimate

$$
\eta_{\alpha \beta}^{(-m)}\left(p_{V}\right) \leqslant C_{\alpha \beta} \eta_{\alpha \beta}^{(-m)}(V)
$$

Here, $\eta_{\alpha \beta}^{(m)}$ is the seminorm as in (2.2).

(2) A principal symbol $q_{V}$ of $T_{n}^{*} \mathcal{F} V \mathcal{F}^{*} T_{n}$ is given by $q_{V}\left(\eta, \eta^{*}\right)=V\left(b^{-1}(\eta),-\eta^{*}\right)$, and moreover, for any $\alpha, \beta \in \mathbf{N}$, there exists $C_{\alpha \beta}>0$ such that the remainder estimates

$$
\eta_{\alpha \beta}^{(-m-1)}\left(p_{V}-q_{V}\right) \leqslant C_{\alpha \beta} \eta_{\alpha \beta}^{(-m-1)}(\nabla V)
$$

holds. Here, we denote by $\nabla V$ the first order derivatives of $V$.

(3) The operator $T_{i}^{*} \mathcal{F} V \mathcal{F}^{*} T_{j}$ on $L^{2}(\mathbf{R})$ belongs to $O p S^{-m-1}$ for any $i, j \in \mathbf{N}$ satisfying $i \neq j$, and moreover, for any $\alpha, \beta \in \mathbf{N}$, there exists $C_{\alpha \beta}>0$ such that the Weyl symbol $r_{V}$ of $T_{i}^{*} \mathcal{F} V \mathcal{F}^{*} T_{j}$ on $L^{2}(\mathbf{R})$ satisfies the estimate

$$
\eta_{\alpha \beta}^{(-m-1)}\left(r_{V}\right) \leqslant C_{\alpha \beta} \eta_{\alpha \beta}^{(-m-1)}(\nabla V)
$$

Proof: First, assuming further that $V \in \mathcal{S}\left(\mathbf{R}^{2}\right)$, we show the assertions (1)-(3). For any $i, j$ $\in \mathbf{N}$, we find that the operator $T_{i}^{*} \mathcal{F} V \mathcal{F}^{*} T_{j}$ has the integral kernel $K\left(\eta, \eta^{\prime}\right)$ $=\iint_{\mathbf{R}^{2} \varphi_{i}}(x, \eta) \varphi_{j}\left(x, \eta^{\prime}\right) V(x, y) e^{-\sqrt{-1} y\left(\eta-\eta^{\prime}\right)} \mathrm{d} x \bar{d} y$, which converges absolutely for each $\eta, \eta^{\prime}$, and so the Weyl symbol $p_{V}$ is given by

$$
\begin{aligned}
p_{V}\left(\eta, \eta^{*}\right) & =\int_{\mathbf{R}} e^{-\sqrt{-1} w \eta^{*}} K(\eta+w / 2, \eta-w / 2) \mathrm{d} w \\
& =\iiint_{\mathrm{R}^{3}} \mathrm{~d} x d w \mathrm{~d} z e^{-\sqrt{-1} w z} \varphi_{i}(x, \eta+w / 2) \varphi_{j}(x, \eta-w / 2) V\left(x, z-\eta^{*}\right),
\end{aligned}
$$

where we changed the variable $z=y+\eta^{*}$ in the last line. An integration by parts yields that the rhs of (5.5) is equal to

$$
\iiint_{\mathbf{R}^{3}} \mathrm{~d} x \mathrm{~d} w \mathrm{~d} z\langle z\rangle^{-2 N} e^{-\sqrt{-1} w z}\left\langle D_{w}\right\rangle^{2 N}\left(\varphi_{n}(x, \eta+w / 2) \varphi_{n}(x, \eta-w / 2)\right) V\left(x, z-\eta^{*}\right)
$$

for any $N \in \mathbf{N}$. Using the estimate $\left\langle\eta ; \eta^{*}\right\rangle \leqslant C\left\langle x ; z-\eta^{*}\right\rangle\langle\eta-b(x)\rangle\langle z\rangle$, we find that, for any $\alpha, \beta$,

$$
\begin{aligned}
& \left\langle\eta ; \eta^{*}\right\rangle^{m+\alpha} \mid \partial_{\eta}^{\beta} \partial_{\eta^{*} p_{V}\left(\eta, \eta^{*}\right) \mid} \\
& \quad \leqslant C \iiint_{\mathbf{R}^{3}} \mathrm{~d} x d w \mathrm{~d} z\langle z\rangle^{-2 N+m+\alpha}\langle\eta-b(x)\rangle^{m+\alpha} \\
& \quad \times\left|\partial_{\eta}^{\beta}\left\langle D_{w}\right\rangle^{2 N}\left(\varphi_{i}(x, \eta+w / 2) \varphi_{j}(x, \eta-w / 2)\right)\right|\left\langle x ; z-\eta^{*}\right\rangle^{m+\alpha} \\
& \quad\left|\partial_{\eta^{*}}^{\alpha} V\left(x, z-\eta^{*}\right)\right|
\end{aligned}
$$




$$
\leqslant C^{\prime} \eta_{\alpha 0}^{(-m)}(V) \iint_{\mathbf{R}^{2}} \mathrm{~d} x \bar{d} w\langle\eta-b(x)\rangle^{m+\alpha}\left|\partial_{\eta}^{\beta}\left\langle D_{w}\right\rangle^{2 N}\left(\varphi_{i}(x, \eta+w / 2) \varphi_{j}(x, \eta-w / 2)\right)\right|
$$

if we take $N$ so that $2 N-m-\alpha>1$. By Lemma 4.7 , we have

$$
\begin{aligned}
& \left|\partial_{\eta}^{\beta}\left\langle D_{w}\right\rangle^{2 l}\left(\varphi_{i}(x, \eta+w / 2) \varphi_{j}(x, \eta-w / 2)\right)\right| \\
& \quad \leqslant C_{\beta N} \sum_{0 \leqslant \beta^{\prime} \leqslant \beta} \sum_{0 \leqslant N^{\prime} \leqslant 2 N}\left|\partial_{\eta}^{\beta-\beta^{\prime}+2 N-N^{\prime}} \varphi_{i}(x, \eta+w / 2) \partial_{\eta}^{\beta^{\prime}+N^{\prime}} \varphi_{j}(x, \eta-w / 2)\right| \\
& \quad \leqslant C_{\beta N}^{\prime} \exp \left(-c\left((b(x)-\eta-w / 2)^{2}-(b(x)-\eta+w / 2)^{2}\right)\right)=C_{\beta N}^{\prime} \exp \left(-2 c(b(x)-\eta)^{2}-c w^{2} / 2\right)
\end{aligned}
$$

Hence, the integral on the rhs of (5.7) converges absolutely, because of Lemma 4.2. Especially, when $i=j=n$, this proves the assertion (1) under the temporal assumption on $V$.

We show the assertions (2) and (3). The first order Taylor expansion yields that

$$
\begin{aligned}
V\left(x, z-\eta^{*}\right)= & V\left(b^{-1}(\eta),-\eta^{*}\right)+\left(x-b^{-1}(\eta)\right) \int_{0}^{1}\left(\partial_{1} V\right)\left(\theta x+(1-\theta) b^{-1}(\eta), \theta\left(z-\eta^{*}\right)-(1-\theta) \eta^{*}\right) \mathrm{d} \theta \\
& +z \int_{0}^{1}\left(\partial_{2} V\right)\left(\theta x+(1-\theta) b^{-1}(\eta), \theta\left(z-\eta^{*}\right)-(1-\theta) \eta^{*}\right) \mathrm{d} \theta
\end{aligned}
$$

where $\partial_{j} V$ denotes the derivative of $V$ with respect to the $j$ th variable. In (5.5), the symbol corresponding to the first term on the rhs of (5.8) is given by

$$
\begin{gathered}
\iiint_{\mathbf{R}^{3}} \mathrm{~d} x \bar{d} w \mathrm{~d} z e^{-\sqrt{-1} w z} \varphi_{i}(x, \eta+w / 2) \varphi_{j}(x, \eta-w / 2) V\left(b^{-1}(\eta),-\eta^{*}\right) \\
=\int_{\mathbf{R}} \varphi_{i}(x, \eta) \varphi_{j}(x, \eta) \mathrm{d} x V\left(b^{-1}(\eta),-\eta^{*}\right)=V\left(b^{-1}(\eta),-\eta^{*}\right) \delta_{i j},
\end{gathered}
$$

where we used the Fourier inversion formula. We can derive the estimate (5.3) for the symbols corresponding to the second and the third terms on the rhs of (5.8) in the same way as in the proof of (5.2), using the facts that $\left|\partial_{\eta}^{\alpha}\left(x-b^{-1}(\eta)\right)\right| \leqslant C_{\alpha}\left\langle x-b^{-1}(\eta)\right\rangle$, which follows from (B.2), and that $\left\langle\eta ; \eta^{*}\right\rangle \leqslant C\left\langle b^{-1}(\eta) ;-\eta^{*}\right\rangle \leqslant C^{\prime}\left\langle\theta x+(1-\theta) b^{-1}(\eta) ; \theta\left(z-\eta^{*}\right)-(1-\theta) \eta^{*}\right\rangle\left\langle x-b^{-1}(\eta)\right\rangle\langle z\rangle$. Then we have the assertions (2) and (3) when $V \in \mathcal{S}\left(\mathbf{R}^{2}\right)$.

Finally, we give a proof for general $V \mathrm{~s}$. We consider only the case of $i=j=n$, since the case of $i \neq j$ is similar. Since the space $\mathcal{S}(\mathbf{R})$ is dense in $S^{-k}$ for any $k>0$, we can approximate $V$ by an appropriate sequence $\left\{V_{l}\right\}_{l=1}^{\infty}\left(\subset \mathcal{S}\left(\mathbf{R}^{2}\right)\right)$ in $S^{-k}$ for any $k>0$. We consider the equality

$$
T_{n}^{*} \mathcal{F} V_{l} \mathcal{F}^{*} T_{n}=O p\left(q_{V_{1}}\right)+R\left(\nabla V_{l}\right)
$$

where $R\left(\nabla V_{l}\right)$ stands for the remainder term. Then it follows from Lemma 2.5, (5.2) and (5.3) that the rhs of (5.9) converges to $O p\left(q_{V}\right)+R(\nabla V)$ as $l \rightarrow \infty$ in the norm operator topology. On the other hand, the left-hand side (lhs) of (5.9) converges to $T_{n}^{*} \mathcal{F} V \mathcal{F}^{*} T_{n}$ as $l \rightarrow \infty$ in the weak operator topology by definition. Thus, the lemma is true for general $V$ 's.

The next lemma follows immediately from Lemma 5.1 if we replace $V$ by $V^{\alpha}$.

Lemma 5.2: Let $\mathcal{V}$ be the operator (5.1) with $W=V$. Let $\alpha>0$. Set $\mathcal{V}_{i j}^{\alpha}=T_{i} \mathcal{F} V^{\alpha} \mathcal{F}^{*} T_{j}$ for any $i, j \in \mathbf{N}$. If we regard $\mathcal{V}_{i j}^{\alpha}$ as an operator from the $j$ th component to the $i$ th component of $\Sigma_{l \in \mathbf{N}}$ $\oplus L^{2}(\mathbf{R})$, then $\mathcal{V}_{i j}^{\alpha}$ is a $\Psi$ DO whose symbol is given by the form $V\left(b^{-1}(\eta),-\eta^{*}\right) \delta_{i j}+O p S^{-m \alpha-1}$.

We need the following result in the place where we use Proposition 2.8.

Lemma 5.3: Let $\lambda \in \mathbf{R} \backslash \operatorname{Spec}\left(H_{0}(b)\right)$ and let $\nu_{l}$ be as in Sec. I. For any $l \in \mathbf{N}$, we have $\lim _{g \rightarrow \infty} g^{-2 / m} \operatorname{Vol}\left\{(x, \xi) \in \mathbf{R}^{2}\left|V\left(b^{-1}(x),-\xi\right)>g^{-1}\right| \lambda_{l}(x)-\lambda \mid\right\}=\nu_{l}(\lambda)$.

Proof: Note that $\left|\lambda_{l}(b(x))-\lambda\right| \geqslant C_{\lambda}>0$ holds for some $C_{\lambda}$, independent of $l, x$. For simplicity, 
we set $F_{l}=\left\{(x, \xi) \in \mathbf{R}^{2}\left|V\left(b^{-1}(x),-\xi\right)>g^{-1}\right| \lambda_{l}(x)-\lambda \mid\right\}, F_{l}^{\prime}=\left\{(x, \xi) \in \mathbf{R}^{2}\left|V(x, \xi)>g^{-1}\right| \lambda_{l}(b(x))-\lambda \mid\right\}$ for any $l \in \mathbf{N}$. By changing the variables $(x, \xi) \rightarrow(b(x),-\xi)$, we find that $(1 / 2 \pi) \operatorname{Vol}\left(F_{l}\right)$ $=\iint_{F_{l}^{\prime}} B(x) \mathrm{d} x d \xi=J_{0}+J_{+}+J_{-}$, where we set

$$
J_{0}=\int_{F_{l}^{\prime} \cap\left\{|x| \leqslant g^{1 / 2 m}\right\}} B(x) \mathrm{d} x d \xi, \quad J_{ \pm}=\iint_{F_{l}^{\prime} \cap\left\{ \pm x \geqslant g^{1 / 2 m}\right\}} B(x) \mathrm{d} x d \xi,
$$

respectively. We first consider the integral $J_{0}$. By $(B .1),(V .1)$, there exists $C>0$ such that $J_{0}$ $\leqslant B_{+} \operatorname{Vol}\left\{(x, \xi) \in \mathbf{R}^{2}\left|C\langle x, \xi\rangle^{-m}>C_{\lambda} g^{-1},\right| x \mid \leqslant g^{1 / 2 m}\right\}=o\left(g^{2 / m}\right)$ holds as $g \rightarrow \infty$. Next, we consider the integral $J_{+}$. We divide $J_{+}=J_{+, 1}+J_{+, 2}$ with

$$
\begin{gathered}
J_{+, 1}=\int_{F_{l}^{\prime} \cap\left\{x \geqslant g^{1 / 2 m\}}\right.}\left(B(x)-B_{+}\right) \mathrm{d} x d \xi, \\
J_{+, 2}=\frac{B_{+}}{2 \pi} \operatorname{Vol}\left\{(x, \xi) \in \mathbf{R}^{2}\left|V(x, \xi)>g^{-1}\right| \lambda_{l}(b(x))-\lambda \mid, x \geqslant g^{1 / 2 m}\right\} .
\end{gathered}
$$

Using (B.2) we find that $\left|J_{+, 1}\right|$ is bounded from above by

$$
C \int_{F_{l}^{\prime} \cap\left\{x \geqslant g^{1 / 2 m}\right\}}\langle x\rangle^{-M} \mathrm{~d} x \vec{d} \xi \leqslant C g^{-M / 2 m} \operatorname{Vol}\left\{(x, \xi) \in \mathbf{R}^{2} \mid\langle x ; \xi\rangle^{-m}>C_{\lambda} g^{-1}, x \geqslant g^{1 / 2 m}\right\},
$$

which is of order $o\left(g^{2} / m\right)$ as $g \rightarrow \infty$. If $x \leqslant g^{1 / 2 m}$, then $\left|\lambda_{l}(b(x))-\lambda\right| \geqslant\left|\Lambda_{l}^{+}-\lambda\right|-\left|\lambda_{l}(b(x))-\Lambda_{l}^{+}\right|$ $\geqslant\left|\Lambda_{l}^{+}-\lambda\right|-C\langle x\rangle^{-M} \geqslant\left|\Lambda_{l}^{+}-\lambda\right|-C g^{-M / 2 m}$ holds because of Lemma 4.8. Then we find that $J_{+, 2}$ $=\left(B_{+} / 4 \pi\right)\left|\Lambda_{l}^{+}-\lambda\right|^{-2 / m} \hat{v}+g^{2 / m}+o\left(g^{2 / m}\right)$ as $g \rightarrow \infty$ in the same way as in the proof of Lemma 3.6. We can estimate the integral $J_{-}$similarly.

\section{B. Preliminary estimates}

The proofs of Theorems 1.1, 1.4, and 1.5 given below are essentially the same as those of Theorems 1.2, 1.1 in Raikov (1993) and that of Theorem 2.2 in Raikov (1998), respectively. However, we reproduce the proofs of Theorems 1.1-1.5 for the sake of completeness.

Let $\Lambda_{n-1}^{+}<\lambda<\Lambda_{n}^{-}$. Take an integer $N_{0}$ greater than $n$ and set $I_{-}=\{-1,0, \ldots, n-1\}, I_{+}=\{n, n$ $\left.+1, \ldots, N_{0}\right\}$. Define the orthogonal projections $P_{+}, P_{-}, P_{\infty}$, and $P_{-}^{c}$ on the space $\Sigma_{l \in \mathbf{N}} \oplus L^{2}(\mathbf{R})$ by $P_{ \pm}=\Sigma_{l \in I_{+}} \oplus \mathrm{id} L^{2}(\mathbf{R})$, respectively, $P_{\infty}=\mathrm{id}-P_{-}-P_{+}$and $P_{-}^{c}=P_{+}+P_{\infty}=\mathrm{id}-P_{-}$. Here, "id" stands for the identity operator. These projections $P_{ \pm}, P_{\infty}$ commute with each other and with the operator $\mathcal{H}_{0}$.

Lemma 5.4: We have the asymptotic relations

$$
\begin{gathered}
\lim _{g \rightarrow \infty} g^{-2 / m} N\left(g^{-1}<\mathcal{V}^{1 / 2} P_{ \pm}\left|\mathcal{H}_{0}-\lambda\right|^{-1} \mathcal{V}^{1 / 2}\right)=\sum_{l \in I_{ \pm}} \nu_{l}(\lambda), \\
\lim _{g \rightarrow \infty} g^{-2 / m} N\left(g^{-1}<P_{ \pm} \mathcal{V}^{1 / 2} P_{ \pm}\left|\mathcal{H}_{0}-\lambda\right|^{-1} \mathcal{V}^{1 / 2} P_{ \pm}\right)=\sum_{l \in I_{ \pm}} \nu_{l}(\lambda),
\end{gathered}
$$

respectively. Here, the operators $\mathcal{H}_{0}$ and $\mathcal{V}$ are as in Sec. V A.

Proof: It follows from $\operatorname{Spec}\left(A^{*} A\right) \backslash\{0\}=\operatorname{Spec}\left(A A^{*}\right) \backslash\{0\}$ that

$$
N\left(g^{-1}<\mathcal{V}^{1 / 2} P_{ \pm}\left|\mathcal{H}_{0}-\lambda\right|^{-1} \mathcal{V}^{1 / 2}\right)=N\left(g^{-1}<\left|\mathcal{H}_{0}-\lambda\right|^{-1 / 2} P_{ \pm} \mathcal{V} P_{ \pm}\left|\mathcal{H}_{0}-\lambda\right|^{-1 / 2}\right) .
$$

The operators $\left|\mathcal{H}_{0}-\lambda\right|^{-1} P_{ \pm}$are matrix-valued $\Psi$ DOs on $\Sigma_{l \in I_{ \pm}} \oplus L^{2}(\mathbf{R})$ and have the symbol $\left(\left|\lambda_{l}(\eta)-\lambda\right|^{-1} \delta_{i j}\right)_{i, j \in I_{+}}$, which belong to the class $O p S^{0}$ by Lemma 4.5 , and the operators $\mid \mathcal{H}_{0}$ $-\left.\lambda\right|^{-1 / 2} P_{ \pm} \mathcal{V} P_{ \pm}\left|\mathcal{H}_{0}-\bar{\lambda}\right|^{-1 / 2}$ are matrix-valued $\Psi$ DOs whose principal symbols are given by $\left(\mid \lambda_{l}(\eta)\right.$ 
$\left.-\left.\lambda\right|^{-1} V\left(b^{-1}(\eta), \eta^{*}\right) \delta_{i j}\right)_{i, j \in I_{ \pm}}$, respectively, because of Lemma 5.2. Then (5.10) follows from a matrix-valued $\Psi D O$ version of Proposition 2.8. The proof of (5.11) is similar.

Lemma 5.5: We have the asymptotic relations

$$
\begin{gathered}
\lim _{\mu \downarrow 0} \mu^{2 / m} N\left(\mu<P_{-}^{c} \mathcal{V}^{1 / 2} P_{-}\left|\mathcal{H}_{0}-\lambda\right|^{-1} \mathcal{V}^{1 / 2} P_{-}^{c}\right)=0, \\
\lim _{\mu \downarrow 0} \mu^{2 / m} N\left(\mu<P_{-} \mathcal{V}^{1 / 2} P_{+}\left|\mathcal{H}_{0}-\lambda\right|^{-1} \mathcal{V}^{1 / 2} P_{-}\right)=0, \\
\lim _{N_{0} \rightarrow \infty} \limsup _{\mu \downarrow 0} \mu^{2 / m} N\left(\mu<P_{-} \mathcal{V}^{1 / 2} P_{\infty}\left|\mathcal{H}_{0}-\lambda\right|^{-1} \mathcal{V}^{1 / 2} P_{-}\right)=0 .
\end{gathered}
$$

Proof: Since $P_{-} \mathcal{V}^{1 / 2} P_{-}^{c} \mathcal{V}^{1 / 2} P_{-}=P_{-} \mathcal{V}^{1 / 2}\left(\mathrm{id}-P_{-}\right) \mathcal{V}^{1 / 2} P_{-}=P_{-} \mathcal{V}_{-} P_{-}\left(P_{-} \mathcal{V}^{1 / 2} P_{-}\right)^{2}$, we can deduce from Lemma 5.2 that $P_{-} \mathcal{V}^{1 / 2} P_{-}^{c} \mathcal{V}^{1 / 2} P_{-}$belongs to $O p S^{-m-1}$. Then it follows from (2.4) that $N(\mu$ $\left.<\left|\mathcal{H}_{0}-\lambda\right|^{-1 / 2} P_{-} \mathcal{V}^{1 / 2} P_{-}^{c} \mathcal{V}^{1 / 2} P_{-}\left|\mathcal{H}_{0}-\lambda\right|^{-1 / 2}\right)=O\left(\mu^{-2 /(m+1)}\right)=o\left(\mu^{-2 /(m+1)}\right)$ as $\mu \downarrow 0$. This proves (5.12) since $\operatorname{Spec}\left(A^{*} A\right) \backslash\{0\}=\operatorname{Spec}\left(A A^{*}\right) \backslash\{0\}$.

The operator $P_{-} \mathcal{V}^{1 / 2} P_{+}$belongs to $O p S^{-m / 2-1}$ by Lemma 5.2 since $\Sigma_{l \in I_{-}} \oplus L^{2}(\mathbf{R})$ and $\Sigma_{l \in I_{+}}$ $\oplus L^{2}(\mathbf{R})$ are orthogonal, and $P_{+} \mathcal{V}^{1 / 2} P_{-} \mathcal{V}^{1 / 2} P_{+}=\left(P_{-} \mathcal{V}^{1 / 2} P_{+}\right)^{*}\left(P_{-} \mathcal{V}^{1 / 2} P_{+}\right)$belongs to $O p S^{-m-2}$. Then (2.4) proves (5.13).

By the definition of $P_{\infty}$ and the min-max argument, we have

$$
N\left(\mu<P_{-} \mathcal{V}^{1 / 2} P_{\infty}\left|\mathcal{H}_{0}-\lambda\right|^{-1} \mathcal{V}^{1 / 2} P_{-}\right) \leqslant N\left(\left(\Lambda_{N_{0}+1}^{-}-\lambda\right)_{\mu}<P_{-} \mathcal{V} P_{-}\right) \leqslant C\left(\Lambda_{N_{0}+1}^{-}-\lambda\right)^{-2 / m} \mu^{-2 / m},
$$

where we used the fact that $P_{-} \mathcal{V} P_{-} \in O p S^{-m}$ and (2.4) in the last inequality. Since $\Lambda_{N_{0+1}}^{-}$tends to infinity as $N_{0} \rightarrow \infty$, we have the conclusion.

Lemma 5.6: We have $\lim _{N_{0} \rightarrow \infty} \lim \sup _{\mu \downarrow 0} \mu^{2 / m} N\left(\mu<\mathcal{V}^{1 / 2} P_{\infty}\left|\mathcal{H}_{0}-\lambda\right|^{-1} \mathcal{V}^{1 / 2}\right)=0$.

Proof: If we choose $N_{0}>0$ so large that $\lambda \leqslant \Lambda_{N_{0}+1}^{-} / 2$ holds, then $\Lambda_{j}(\eta)-\lambda \geqslant \lambda_{j}(\eta)-\Lambda_{N_{0}+1}^{-} / 2$ $\geqslant\left(\lambda_{j}(\eta)+\Lambda_{N_{0}+1}^{-}\right) / 4$ holds for any $j \geqslant N_{0}+1$ and for any $\eta \in \mathbf{R}$, from which we have $P_{\infty} \mid \mathcal{H}_{0}$ $-\left.\lambda\right|^{-1} \leqslant 4\left(\mathcal{H}_{0}+\Lambda_{N_{0}+1}^{-}\right)^{-1}$. Then the variational principle yields the estimate $N\left(\mu<\mathcal{V}^{1 / 2} P_{\infty} \mid \mathcal{H}_{0}\right.$ $\left.-\left.\lambda\right|^{-1} \mathcal{V}^{1 / 2}\right) \leqslant N\left(\mu<4 \mathcal{V}^{1 / 2}\left(\mathcal{H}_{0}+\Lambda_{N_{0}+1}^{-}\right)^{-1} \mathcal{V}^{1 / 2}\right)=N\left(H_{4 / \mu}(b)<-\Lambda_{N_{0}+1}\right)=\Sigma_{l \in \mathbf{N}} \nu_{l}\left(-\Lambda_{N_{0}+1}^{-}\right)(4 / \mu)^{2 / m}(1$ $+o(1))$ as $\mu \downarrow 0$, where we used Theorem 1.1 proved in Sec. III in the last inequality. Finally, the lemma follows from the asymptotic relation $\lim _{N_{0} \rightarrow \infty} \Sigma_{l \in \mathbf{N}} \nu_{l}\left(-\Lambda_{N_{0}+1}^{-}\right)=0$.

\section{Proof of Theorem 1.1}

Using the inequality $\mathcal{V}^{1 / 2}\left(\mathcal{H}_{0}-\lambda\right)^{-1} \mathcal{V}^{1 / 2} \leqslant \mathcal{V}^{1 / 2} P_{-}^{c}\left|\mathcal{H}_{0}-\lambda\right|^{-1} \mathcal{V}^{1 / 2}$, we have, for any small $\varepsilon>0$,

$$
\begin{aligned}
N_{g}^{+}(\lambda) \leqslant & N\left(g^{-1}<\mathcal{V}^{1 / 2} P_{-}^{c}\left|\mathcal{H}_{0}-\lambda\right|^{-1} \mathcal{V}^{1 / 2}\right) \leqslant N\left((1-\varepsilon) g^{-1}<\mathcal{V}^{1 / 2} P_{+}\left|\mathcal{H}_{0}-\lambda\right|^{-1} \mathcal{V}^{1 / 2}\right) \\
& +N\left(\varepsilon g^{-1}<\mathcal{V}^{1 / 2} P_{\infty}\left|\mathcal{H}_{0}-\lambda\right|^{-1} \mathcal{V}^{1 / 2}\right),
\end{aligned}
$$

where we used the (generalized) Birman-Schwinger principle [see, e.g., Alama, Deift, and Hempel (1989), Birman (1991)] and the Weyl-KyFan inequality. Then by Lemma 5.4 and Lemma 5.6, we obtain the upper bound $\lim \sup _{g \rightarrow \infty} g^{-2 / m} N_{g}^{+}(\lambda) \leqslant \Sigma_{l \in I_{+}} \nu_{l}(\lambda)$, taking a limit $\varepsilon \downarrow 0$ and $N_{0}$ $\rightarrow \infty$. Next, we obtain the lower bound. For any small $\varepsilon>0$, we have

$$
\begin{aligned}
P_{-}^{c} \mathcal{V}^{1 / 2}\left(\mathcal{H}_{0}-\lambda\right)^{-1} \mathcal{V}^{1 / 2} P_{-}^{c}= & P_{-}^{c} \mathcal{V}^{1 / 2} P_{-}\left(\mathcal{H}_{0}-\lambda\right)^{-1} \mathcal{V}^{1 / 2} P_{-}^{c}+P_{-}^{c} \mathcal{V}^{1 / 2} P_{-}^{c}\left(\mathcal{H}_{0}-\lambda\right)^{-1} \mathcal{V}^{1 / 2} P_{-}^{c} \\
\geqslant & P_{-}^{c} \mathcal{V}^{1 / 2} P_{-}\left(\mathcal{H}_{0}-\lambda\right)^{-1} \mathcal{V}^{1 / 2} P_{-}^{c}+\mathcal{V}^{1 / 2} P_{-}^{c}\left(\mathcal{H}_{0}-\lambda\right)^{-1} \mathcal{V}^{1 / 2} \\
& -2 \operatorname{Re}\left(P_{-} \mathcal{V}^{1 / 2} P_{-}^{c}\left(\mathcal{H}_{0}-\lambda\right)^{-1} \mathcal{V}^{1 / 2}\right) \geqslant(1-\varepsilon) \mathcal{V}^{1 / 2} P_{-}^{c}\left|\mathcal{H}_{0}-\lambda\right|^{-1} \mathcal{V}^{1 / 2} \\
& -P_{-}^{c} \mathcal{V}^{1 / 2} P_{-}\left|\mathcal{H}_{0}-\lambda\right|^{-1} \mathcal{V}^{1 / 2} P_{-}^{c}-\varepsilon^{-1} P_{-} \mathcal{V}^{1 / 2} P_{-}^{c}\left|\mathcal{H}_{0}-\lambda\right|^{-1} \mathcal{V}^{1 / 2} P_{-},
\end{aligned}
$$

where we used the inequality $P_{-} \mathcal{V}^{1 / 2} P_{-}^{c}\left(\mathcal{H}_{0}-\lambda\right)^{-1} \mathcal{V}^{1 / 2} P_{-} \geqslant 0$ in the first inequality and used the estimate $2\left|\left(u, \mathcal{V}^{1 / 2} P_{-}^{c}\left(\mathcal{H}_{0}-\lambda\right)^{-1} \mathcal{V}^{1 / 2} P_{-} u\right)\right| \leqslant \varepsilon\left\|\left|\mathcal{H}_{0}-\lambda\right|^{-1 / 2} P_{-}^{c} \mathcal{V}^{1 / 2} u\right\|^{2}+\varepsilon^{-1}\left\|P_{-}^{c}\left|\mathcal{H}_{0}-\lambda\right|^{-1 / 2} \mathcal{V}^{1 / 2} P_{-} u\right\|^{2}$ in 
the second inequality. Then, by a variational argument similar to that used in the case of the upper bound, we can derive the lower bound $\liminf _{g \rightarrow \infty} g^{-2 / m} N_{g}^{+}(\lambda) \geqslant \Sigma_{l \in I_{+}} \nu_{l}(\lambda)$, using Lemma 5.4 and Lemma 5.5. This completes the proof of Theorem 1.1.

\section{Proof of Theorem 1.4}

By the Birman-Schwinger principle, we have the upper bound

$$
\begin{aligned}
N_{g}(\lambda) & =N\left(g^{-1}<-\mathcal{V}^{1 / 2}\left(\mathcal{H}_{0}-\lambda\right)^{-1} \mathcal{V}^{1 / 2}\right) \leqslant N\left(g^{-1}<\mathcal{V}^{1 / 2} P_{-}\left|\mathcal{H}_{0}-\lambda\right|^{-1} \mathcal{V}^{1 / 2}\right) \\
& \leqslant \sum_{l \in I_{-}} \nu_{l}(\lambda) g^{-2 / m}(1+o(1))
\end{aligned}
$$

as $g \rightarrow \infty$, where we used the inequality $-\mathcal{V}^{1 / 2}\left(\mathcal{H}_{0}-\lambda\right)^{-1} \mathcal{V}^{1 / 2}=\mathcal{V}^{1 / 2} P_{-}\left|\mathcal{H}_{0}-\lambda\right|^{-1} \mathcal{V}^{1 / 2}-\mathcal{V}^{1 / 2} P_{-}^{c} \mid \mathcal{H}_{0}$ $-\left.\lambda\right|^{-1} \mathcal{V}^{1 / 2} \leqslant \mathcal{V}^{1 / 2} P_{-}\left|\mathcal{H}_{0}-\lambda\right|^{-1} \mathcal{V}^{1 / 2}$ in the first inequality and used (5.10) in the last inequality. Next, for any small $\varepsilon>0$, there exists $C_{\varepsilon}>0$, independent of $g, N_{0}$, such that

$$
\begin{aligned}
N_{g}^{-}(\lambda) \geqslant & N\left(g^{-1}<-P_{-} \mathcal{V}^{1 / 2}\left(\mathcal{H}_{0}-\lambda\right)^{-1} \mathcal{V}^{1 / 2} P_{-}\right) \geqslant N\left((1+\varepsilon) g^{-1}<P_{-} \mathcal{V}^{1 / 2} P_{-}\left|\mathcal{H}_{0}-\lambda\right|^{-1} \mathcal{V}^{1 / 2} P_{-}\right) \\
& -N\left(C_{\varepsilon} g^{-1}<P_{-} \mathcal{V}^{1 / 2} P_{-}^{c}\left|\mathcal{H}_{0}-\lambda\right|^{-1} \mathcal{V}^{1 / 2} P_{-}\right) \geqslant N\left((1+\varepsilon) g^{-1}<P_{-} \mathcal{V}^{1 / 2} P_{-}\left|\mathcal{H}_{0}-\lambda\right|^{-1} \mathcal{V}^{1 / 2} P_{-}\right) \\
& -N\left(C_{\varepsilon}^{\prime} g^{-1}<P_{-} \mathcal{V}^{1 / 2} P_{+}\left|\mathcal{H}_{0}-\lambda\right|^{-1} \mathcal{V}^{1 / 2} P_{-}\right)-N\left(C_{\varepsilon}^{\prime} g^{-1}<P_{-} \mathcal{V}^{1 / 2} P_{\infty}\left|\mathcal{H}_{0}-\lambda\right|^{-1} \mathcal{V}^{1 / 2} P_{-}\right) \\
\geqslant & \sum_{l \in I_{-}} \nu_{l}(\lambda)(g /(1+\varepsilon))^{2 / m}(1+o(1))
\end{aligned}
$$

as $g, N_{0} \rightarrow \infty$, where we used the Weyl-KyFan inequality in the second and the third inequalities and Lemma 5.4 in the last line. This gives the lower bound and we complete the proof.

\section{E. Proof of Theorem 1.5}

Let $\Lambda_{n-1}^{+}<\lambda<\mu<\Lambda_{n}^{-}$. Set $\gamma=(\mu+\lambda) / 2, \tau=(\mu-\lambda) / 2$ and set $P_{+}^{c}=P_{-}+P_{\infty}$. Since $P_{+}\left(\mathcal{H}_{g}\right.$ $-\gamma)^{2} P_{+}=\left(P_{+}\left(\mathcal{H}_{g}-\gamma\right) P_{+}\right)^{2}-g^{2} P_{+} \mathcal{V} P_{+}^{c} \mathcal{V} P_{+}$, we have, for small $\varepsilon>0, N\left(\lambda \leqslant H_{g}(b)<\mu\right)$ $\geqslant N\left(\left(\mathcal{H}_{g}-\gamma\right)^{2}<\tau^{2}\right) \geqslant N\left(P_{+}\left(\mathcal{H}_{g}-\gamma\right)^{2} P_{+}<\tau^{2}\right) \geqslant N\left(\left(P_{+}\left(\mathcal{H}_{g}-\gamma\right) P_{+}\right)^{2}<\tau^{2}-\varepsilon\right)-N\left(C_{\varepsilon}<g^{2} P_{+} \mathcal{V} P_{+}^{c} \mathcal{V} P_{+}\right)$ $=N\left(\left(P_{+}\left(\mathcal{H}_{g}-\gamma\right) P_{+}\right)^{2}<\tau^{2}-\varepsilon\right)+o\left(g^{2 / m}\right)$ as $g \rightarrow \infty$. Here, we used the fact that the operator $P_{+} \mathcal{V} P_{+}^{c} \mathcal{V} P_{+}=P_{+} \mathcal{V}^{2} P_{+}-\left(P_{+} \mathcal{V} P_{+}\right)^{2}$ is a matrix-valued $\Psi$ DO of the class $O p S^{-2 m-1}$. Setting $\lambda_{1}=\gamma$ $-\sqrt{\tau^{2}-\varepsilon}$ and $\mu_{1}=\gamma+\sqrt{\tau^{2}-\varepsilon}$, we have $N\left(\lambda_{1}<P_{+} \mathcal{H}_{g} P_{+}<\mu_{1}\right) \geqslant N\left(g^{-1}<\mathcal{V}^{1 / 2} P_{+}\left(\mathcal{H}_{0}-\mu_{1}\right)^{-1} P_{+} \mathcal{V}^{1 / 2}\right)$ $-N\left(g^{-1}<\mathcal{V}^{1 / 2} P_{+}\left(\mathcal{H}_{0}-\lambda_{1}\right)^{-1} P_{+} \mathcal{V}^{1 / 2}\right)=\Sigma_{l \in I_{+}} \nu_{l}\left(\left[\lambda_{1}, \mu_{1}\right]\right) g^{2 / m}(1+o(1))$ as $g \rightarrow \infty$, where we used the Birman-Schwinger principle and Theorem 1.1. Finally, we have Theorem 1.5 from these, letting $\varepsilon \downarrow 0$ and $N_{0} \rightarrow \infty$.

${ }^{1}$ Alama, S., Deift, P. A., and Hempel, R., "Eigenvalue branches of the Schrödinger operator $H-\lambda W$ in a gap of $\sigma(H), "$ Commun. Math. Phys. 121, 291-321 (1989).

${ }^{2}$ Avron, J., Herbst, I., and Simon, B., "Schrödinger operators with magnetic fields I. General interactions," Duke Math. J. 45, 847-883 (1978)

${ }^{3}$ Beals, R., “A general calculus of pseudodifferential operators," Duke Math. J. 42, 1-42 (1975).

${ }^{4}$ Birman, M. S., "Discrete spectrum in the gaps of a continuous one for perturbations with large coupling constant," Adv. Sov. Math. 7, 57-73 (1991).

${ }^{5}$ Colin de Verdiere, Y., "L'asymptotique de Weyl pour les bouteilles magnétiques," Commun. Math. Phys. 105, 327-335 (1986).

${ }^{6}$ Cycon, H. L., Froese, R. G., Kirsch, W., and Simon, B., Schrödinger Operators (Springer-Verlag, New York, 1987)

${ }^{7}$ Dauge, M. and Robert, D., "Weyl's formula for a class of pseudodifferential operators with negative order on $L^{2}\left(\mathbf{R}^{n}\right)$," Lect. Notes Math. 1256, 91-122 (1987).

${ }^{8}$ Exner, P. and Kovařik, H., "Magnetic strip waveguides," J. Phys. A 33, 3297-3311 (2000).

${ }^{9}$ Hempel, R. and Levendorskiı̆, S. Z., "On the eigenvalues in gaps for perturbed magnetic Schrödinger operators," J. Math. Phys. 39, 63-78 (1998).

${ }^{10}$ Hörmander, L., "The Weyl calculus of pseudo-differential operators," Commun. Pure Appl. Math. 32, 359-443 (1979).

${ }^{11}$ Iwatsuka, A., "Examples of absolutely continuous Schrödinger operators in magnetic fields," Publ. Res. Inst. Math. Sci. 21, 385-401 (1985).

${ }^{12}$ Levendorskiı̌, S. L., "Lower bounds for the number of eigenvalue branches for the Schrödinger operator $H-\lambda W$ in a gap of $H$ : the case of indefinite W," Commun. Partial Differ. Equ. 20, 827-854 (1995). 
${ }^{13}$ Levendorskiı̌, S. L., "The asymptotics for the number of eigenvalue branches for the magnetic Schrödinger operator $H-\lambda W$ in a gap of $H$," Math. Z. 223, 609-625 (1996).

${ }^{14}$ Mantoiu, M. and Purice, R., "Some propagation properties of the Iwatsuka model," Commun. Math. Phys. 188, 691-708 (1997).

${ }^{15}$ Raikov, G. D., "Strong electric field eigenvalue asymptotics for the Schrödinger operator with electromagnetic potential," Lett. Math. Phys. 21, 41-49 (1991).

${ }^{16}$ Raikov, G. D., "Strong-electric-field eigenvalue asymptotics for the perturbed magnetic Schrödinger operator," Commun. Math. Phys. 155, 415-428 (1993).

${ }^{17}$ Raikov, G. D., "Asymptotic bounds on the number of the eigenvalues in the gaps of the 2D magnetic Schrödinger operator," Asymptotic Anal. 16, 87-98 (1998).

${ }^{18}$ Reed, M. and Simon, B., Methods of Modern Mathematical Physics (Academic, New York, 1978), Vols. I-IV.

${ }^{19}$ Robert, D., "Propriétés spectrales d'opérateurs pseudo-différentiels," Commun. Partial Differ. Equ. 3, 755-826 (1978).

${ }^{20}$ Shirai, S., "Eigenvalue asymptotics for the Schrödinger operator with steplike magnetic field and decreasing electric potential," Publ. Res. Inst. Math. Sci. 39, 297-330 (2003).

${ }^{21}$ Shubin, M. A., Pseudo-differential Operators and Spectral Theory, Springer Series in Soviet Mathematics (Springer, New York, 1987). 